\title{
Flow regimes in a trapped vortex cell
}

\author{
D. Lasagna · G. Iuso
}

the date of receipt and acceptance should be inserted later

\begin{abstract}
This paper presents results of an experimental investigation on the flow in a trapped vortex cell embedded into a flat plate and interacting with a zeropressure-gradient boundary layer. The objective of the work is to describe the flow features and elucidate some of the governing physical mechanisms, in light of recent investigations on flow separation control using vortex cells. Hot-wire velocity measurements of the shear layer bounding the cell and of the boundary layers upstream and downstream are reported, together with spectral and correlation analyses of wall-pressure fluctuation measurements. Smoke flow visualisations are reported to provide qualitative insight into some relevant features of the internal flow, namely a large-scale flow unsteadiness and possible mechanisms driving the rotation of the vortex core. Results are presented for two very different regimes: a low Reynolds number case where the incoming boundary layer is laminar and its momentum thickness is small compared to the cell opening, and a moderately high Reynolds number case, where the incoming boundary layer is turbulent and the ratio between the momentum thickness and the opening length is significantly larger than in the first case. Implications of the present findings to flow control application of trapped vortex cells are also discussed.
\end{abstract}

D. Lasagna

Engineering and the Environment, University of Southampton, SO17 1BJ, Southampton, United Kingdom, E-mail: davide.lasagna@soton.ac.uk

G. Iuso

Dipartimento di Ingegneria Meccanica e Aerospaziale, Corso Duca degli Abruzzi 24, 10129 Torino, Italy

\section{Introduction}

A trapped vortex cell, further referred to as TVC, is a cavity with a particular shape designed to trap a vortex near the surface of a body. The idea is to achieve a favourable localised interaction with the boundary layer developing on the body, in a region close to flow separation. In certain conditions, the boundary layer can exhibit, downstream of the TVC, higher momentum than without the cell resulting in delayed separation and enhanced performance.

The idea of trapping vortices for separation control was probably first mentioned in Ringleb (1961). Following this work, trapped vortices have been studied for separation control for both external and internal flows, in a variety of industrial and engineering applications. For internal flows, Adkins (1975) demonstrated experimentally that the pressure recovery in a short diffuser could be strongly enhanced by a stabilised trapped vortex. Numerical evidence of effective flow separation control in a 2D diverging diffuser was recently discussed in Mariotti et al (2013) and Mariotti et al (2014).

TVCs have been also employed to control flow separation on lifting bodies to increase aerodynamic performance. Starting from the first implementations, Kasper (1974) and Savitsky et al (1995), several flow control studies have been conducted in the framework of the EU project VortexCell2050, e.g. De Gregorio and Fraioli (2008); Donelli et al (2009b); Olsman et al (2011); Lasagna et al (2011).

Theoretical work, Bunyakin et al (1998); Chernyshenko et al (2003); Zannetti (2006), has shown that a two dimensional trapped vortex flow is usually unstable. A three dimensional instability of the cell flow has been also identified. For example Savelsberg and Castro (2008) and Tutty et al (2012) studied experimentally and the- 
oretically the flow inside a large aspect ratio cylindrical cell, and observed a spanwise modulation of the flow structure due to a natural three-dimensional inviscid instability well predicted by theoretical analysis. A similar spanwise modulation was also observed in a large eddy simulation performed by Hokpunna and Manhart (2007). A three dimensional flow structure was also observed in static pressure measurements inside a cell fitted on a wing with NACA0024 section by Lasagna et al (2011) and embedded in a flate plat in Baranov et al (2000).

In practice, flow instability results in strong unsteadiness, poor recirculation of the cell flow, ejection of vorticity from the cavity and ultimately poor control performance of the TVC as a passive device. In fact, several authors, De Gregorio and Fraioli (2008); Lasagna et al (2011); Vuddagiri and Samad (2013), have demonstrated that the performance of an airfoil equipped with a TVC is generally worse that those of the baseline reference case. Olsman et al (2011) investigated by direct numerical simulation and by flow visualisations in a water tunnel the low Reynolds number flow past a TVC-controlled airfoil. They highlighted a very complex interaction between the structures generated by the cavity shear layer, subject to a Kelvin-Helmoltz instability, and the separated flow downstream of the cavity, which eventually resulted in an increase of the lift-to-drag ratio of the airfoil. Choudhry et al (2016) explored the idea of using a circular cavity as a passive "reservoir" for reverse flow accumulation during the pitch-up phase of dynamic stall of a NACA0021 airfoil, showing a delay in the onset of flow separation.

However, the general indication appears to be that some form of manipulation of the cell flow is necessary to achieve satisfactory global control performance. One possible solution is careful design of the geometry, to reduce or prevent such effects. For example, Chernyshenko et al (2008) used an evolutionary algorithm to search in the space of cavity geometries for that which results in the least internal separation. Shape optimisation, although with different objectives, was also investigated by Mariotti et al $(2013,2014)$ to increase the performance of a diffuser. Nevertheless, the most significant increase in performance have been obtained with active flow control techniques, implemented by several authors, e.g. De Gregorio and Fraioli (2008); Lasagna et al (2011); Tutty et al (2013), typically using steady or unsteady distributed suction in the cavity region to suppress large scale unsteady motion and favour the formation of a stable, coherent and intense trapped vortex. The bottom line of all these investigations is the relevance of unsteady flow phenomena and the ne- cessity of a local active control technique to achieve an overall effectiveness of the TVC as a control strategy.

The main objective of the present paper is to report the results of an experimental investigation focusing specifically on relevant mechanisms and unsteady features of the flow in a trapped vortex cell that are of interest in flow control applications. In order to highlight a broader range of behaviour, we will focus on two different and representative flow regimes, highlighting the different properties that emerge. We have chosen a simplified configuration where a TVC is embedded into a flat plate and interacts with a zero-pressure-gradient boundary layer, similarly to case discussed in Hokpunna and Manhart (2007) and in Donelli et al (2009a). It has been argued, Donelli et al (2009a), Lasagna et al (2011), that the effectiveness of a trapped vortex cell in manipulating the flow over an airfoil is strongly connected to the local interaction with the boundary layer flowing past the cell. These local effects, that may shed light over possible flow control mechanisms on airfoils, can be easily studied on the present configuration, representative of a low-angle-of-attack condition and eliminating possible complex interactions between the cell flow, the separated flow region and the wake dynamics occurring for the airfoil configuration.

\section{Experimental setup}

Experiments were conducted in an open return, blowertype facility, with test section having width and height of 750 and $500 \mathrm{~mm}$, respectively. The effective test section height was reduced to $350 \mathrm{~mm}$ by introducing a higher bottom wall, in order to accommodate the cell in the original test section. Maximum speed in the facility was then about $14 \mathrm{~m} / \mathrm{s}$, at which the turbulence intensity level in the vein of the flow was below $0.5 \%$, mostly associated with low frequency modulations.

A boundary layer suction system was fitted about $400 \mathrm{~mm}$ upstream of the cell cusp. The system consisted of a flush-mounted 120-mm-long perforated plate spanning the entire width of the test section. The plate covered a drawer embedded in the bottom wall, with dividing vanes to distribute suction from an industrial blower. The properties of the boundary layer detaching at the cell cusp, which markedly affect the flow regime in rectangular cavities, e.g. Rockwell and Knisely (1980), were controlled with this system.

A sketch of the cell geometry is reported in figure 1. The reference system adopted and the symbols of the main parameters of the configuration are also shown. The geometry is the same as that for which LES and RANS were performed by Hokpunna and Manhart (2007) and Donelli et al (2009a), respectively. The cav- 


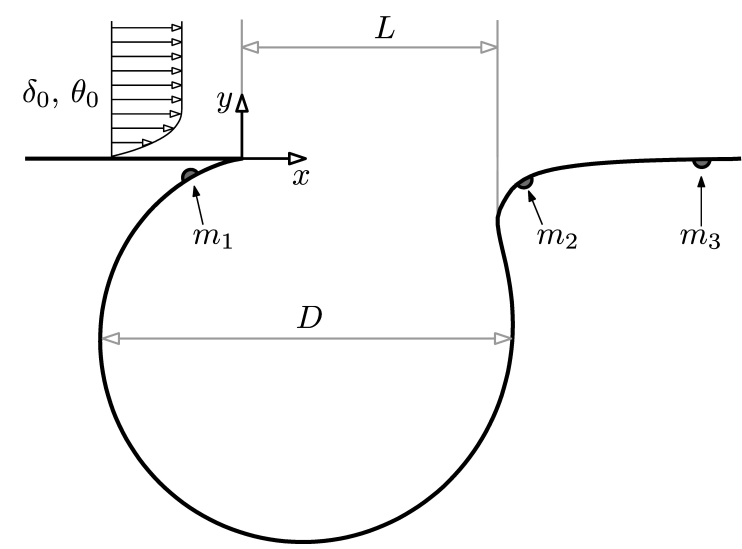

Fig. 1 Sketch of the trapped vortex cell with main geometric parameters, reference system, and location of the microphones for wall-pressure measurements.

ity opening length $L=68 \mathrm{~mm}$ is the projection along the $x$ axis of the cavity opening, $D=114 \mathrm{~mm}$ is a characteristic diameter of the cell, taken as the largest distance between the upstream and downstream faces of the cell. The Reynolds number $R e=u_{e} L / \nu$ is based on the cavity opening length, the velocity in the middle of the vein $u_{e}$ and the kinematic viscosity of air $\nu$ at laboratory temperature. The parameters $\delta_{0}$ and $\theta_{0}$ are the $99 \%$ boundary layer thickness and the momentum thickness of the reference upstream boundary layer, respectively, measured at $x / L=-0.14$, i.e. $x=-10 \mathrm{~mm}$, in the mid-span section. The cavity had a nominal span $b$ of $420 \mathrm{~mm}$, with aspect ratio $b / L$ equal to 6.6 . The cavity spanned $56 \%$ of the test section width, to reduce possible interactions of the cell flow with the boundary layers on the vertical test section walls.

Velocity measurements were performed using an A.A. LabSystems AN1003 constant temperature hot-wire anemometer. The single-wire probe employed, (length 0.9 $\mathrm{mm}$ and diameter $5 \mu \mathrm{m}$ ), was frequently calibrated in situ in the middle of the vein, at a sufficient distance upstream of the cavity against the pressure differential on the contraction section of the facility, measured by a Scanivalve DSA3217 system. A longitudinal slot on the top wall enabled access into the investigation area of the probe holder, moved by a two-axes traverse system allowing fine control of the probe in $(x, y)$ planes.

Wall-pressure fluctuation measurements were performed using high-sensitivity electret condenser microphones, of a similar type as those used by other authors, e.g. Zhang and Naguib (2011); Garcia-Sagrado and Hynes (2011). The microphones, with diameter and height of 9.7 and $6 \mathrm{~mm}$, respectively, were powered by a dedicated power supply unit through in-house-made RC circuits. The frequency response of the microphones was estimated by an ad-hoc calibration experiment fully de- scribed in Lasagna et al (2013). The microphones were mounted in pin-hole configuration, with hole diameter equal to $2 \mathrm{~mm}$.

Results for three relevant microphones, indicated in figure 1 , will be reported. Microphone $m_{1}$ was positioned as close to the cell cusp as the setup allowed. Microphone $m_{2}$ was located on the downstream part of the cell, in the region where the shear layer bounding the cavity impinges on the wall. The last microphone, denoted as $m_{3}$, was located far downstream of the cell, at $x / L=1.8$.

The hot-wire signal was pre-conditioned and then sampled with a 16-bit NI9215 module from National Instruments. The microphones signals did not need any preconditioning and were sampled directly using 24-bit NI9239 modules. All acquisition modules had an internal anti-aliasing filter set at $46 \%$ of the sampling frequency. Signals were simultaneously sampled to allow cross-correlation, cross-spectra and stochastic estimation analyses, by inserting the acquisition modules into a NI cDAQ-9172 chassis.

Smoke flow visualisations were also performed to investigate on the qualitative structure of the internal cell flow, especially where hot-wire measurements would have been too intrusive. A LASER light sheet, with thickness lower than $1 \mathrm{~mm}$, passing through the longitudinal slot on the top of the test section, illuminated the central section of the cell model. Optical access to the investigation area in the cell was provided by Plexiglass lateral walls. A Dantec Dynamics 80 C37 Nanosense MKII $1280 \times 1024$ model camera was used to acquire time-resolved grey-scale images, with sampling rate and exposure time varied for each condition to obtain a good resolution of the internal flow dynamics. Seeding of the flow was performed by injecting smoke directly inside the cavity region, but high-framerate acquisition of the flow field was started after a short transient, to let the flow reach an appropriate concentration of seeding.

\section{Results}

\subsection{Global investigations}

Time histories of pressure fluctuations from the microphones and of velocity in the centreline section of the model, in a region of the shear layer characterised by intense fluctuations, were measured at different free stream speeds $u_{e}$ in the range $2 \div 14 \mathrm{~m} / \mathrm{s}$, at a Reynolds number spanning the range $9000 \div 68000$. The properties of the incoming boundary layer changed across the different experiments, because it was not possible 
to keep them constant as the free stream speed varied. The boundary layer suction pump was always set at the maximum level to obtain the thinnest boundary layer. Hence, the results in this section have a qualitative meaning.

For each free stream speed, the arithmetic average over the samples, denoted with an overbar in what follows, was subtracted from the signal. Power spectral densities of the pressure and velocity fluctuations, further indicated with a prime, were then computed using the Welch algorithm, by averaging spectra from sixteen blocks $2^{15}$ samples long. A Hanning window was also applied, see Bendat and Piersol (1986).

Figure 2 shows the result of this investigation in the form of a free stream velocity/frequency spectrogram for pressure fluctuations from microphone $m 3$. The colour map shows the base ten logarithm of the power spectral density $\Phi$ of the normalised pressure fluctuations $p_{3}^{\prime} / q_{e}$, with $q_{e}=1 / 2 \rho u_{e}^{2}$, where $\rho$ is the laboratory air density.

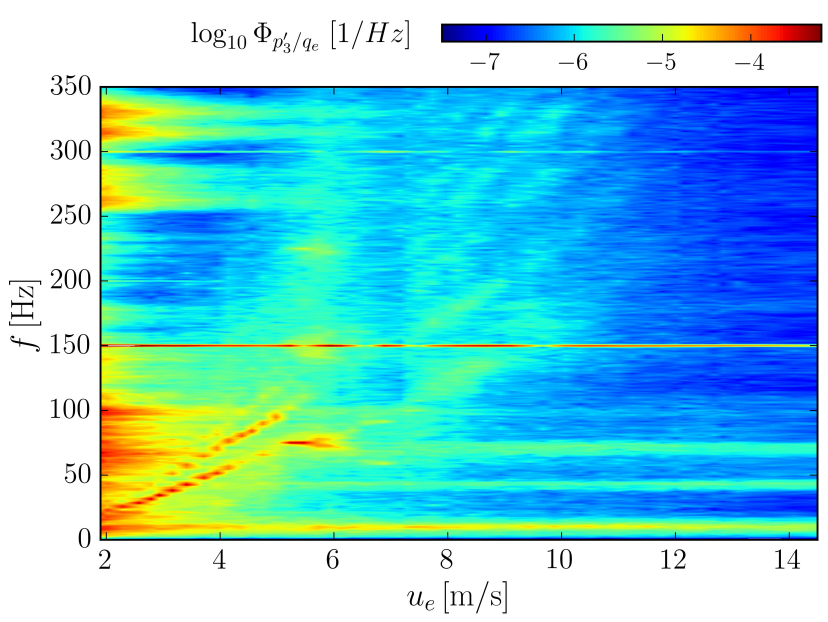

Fig. 2 Free stream velocity/frequency spectrogram of the normalised pressure fluctuations from microphone $m 3$.

For $u_{e} \lesssim 7 \mathrm{~m} / \mathrm{s}$ the incoming boundary layer is laminar. In this range and for frequencies between 25 and $125 \mathrm{~Hz}$, the plot shows three narrow energy concentration bands. These are the signatures of the second, third and fourth Kelvin-Helmoltz instability modes of the shear layer. At low velocities, with laminar incoming boundary layer, the frequencies of these modes, that we will refer to as natural frequencies, are well predicted by the formula for the maximum pulsation frequency in a deep cavity, $f_{n}=0.42 \cdot n u_{e} / L$, Rockwell and Naudascher (1978).

The signature of the first shear layer mode cannot be clearly observed in the spectrogram, even though some energy concentration at the corresponding frequency can be detected by analysis of individual spectral densities. Interestingly, the signature of these modes is still visible for velocities up to about $11 \mathrm{~m} / \mathrm{s}$, for which the incoming boundary layer is already turbulent. The slope of the bands increases slightly with $u_{e}$ likely due to the variation of the boundary layer characteristics across the velocity range.

The horizontal bands, except for that at $150 \mathrm{~Hz}$ and its harmonic at $300 \mathrm{~Hz}$, are due to background noise from the facility and from the boundary layer suction system. However, the strong energy peak at $150 \mathrm{~Hz}$ is due to an acoustic resonance originating in the settling chamber of the facility. This resonance was previously observed in wall-pressure fluctuation measurements on the test section wall without the cavity installed. 2 .

This resonance represents a periodic excitation of the shear layer. At moderate velocities, $u_{e} \lesssim 5 \mathrm{~m} / \mathrm{s}$, the forcing frequency $f_{w t}$ is well separated from the natural frequencies of the laminar shear layer and velocity fluctuations at $f_{w t}$ are not amplified. As the free stream speed increases, a complex interaction between the facility resonance and the shear layer can be observed. At velocities where the frequencies of natural instability modes of the shear layer approach $f_{w t}$, the shear layer locks onto the acoustic resonance and the fluctuation energy at $f_{w t}$ increases significantly, whereas the energy at the natural frequencies is substantially lower. This phenomenon occurs at free stream speeds equal to $5.6,6.5$ and $8.4 \mathrm{~m} / \mathrm{s}$, where lock-in between the fourth, third and second natural shear layer mode occurs, respectively. Lock-in conditions also occur at noninteger frequencies of the wind tunnel resonance mode. At $u_{e} \approx 5 \mathrm{~m} / \mathrm{s}$, the second shear layer mode locks onto the first sub-harmonic of the wind tunnel resonance, at $f=f_{w t} / 2=75 \mathrm{~Hz}$. At slightly higher velocities, two energy bands bifurcate symmetrically with respect to this frequency. A similar interaction, but with lesser intensity, develops at this same velocity for a frequency equal to $f=3 / 2 f_{w t}$.

Figure 3 is the equivalent of figure 2 for the velocity fluctuations. At low speed the signatures of the second and third shear layer modes are clearly visible, while the energy at $f_{w t}$ is quite small, as the shear layer is not locked onto the resonance. However, as the free stream speed increases, the energy of the fluctuations at $f_{w t}$ increases considerably, by several orders of magnitude. Analysis of the individual time histories, not reported here for brevity, highlights a complex interaction between the wind tunnel acoustic resonance and the natural instability modes of the laminar shear layer. For instance, between $5 \mathrm{~m} / \mathrm{s}$ and $6 \mathrm{~m} / \mathrm{s}$ mode competition occurs, with shear layer fluctuations switching intermittently between the natural frequencies and $f_{w t}$. 


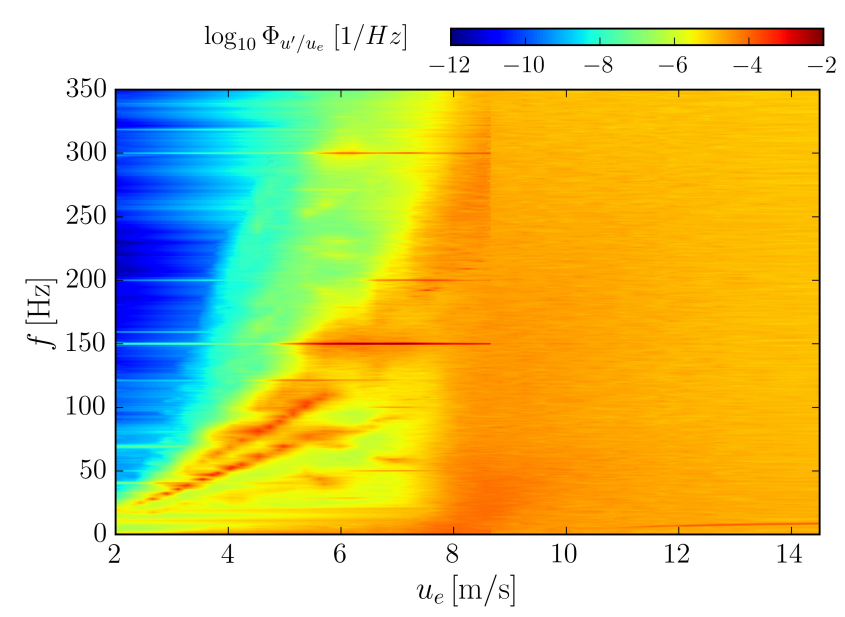

Fig. 3 Free stream velocity/frequency spectrogram of the velocity fluctuations in the shear layer at $x / L=0.58, y / \delta_{0}=$ 0.22 .

For $u_{e}>9 \mathrm{~m} / \mathrm{s}$, no evidence of coherent oscillations of the shear layer flow can be detected. In fact, for $u_{e} \gtrsim$ $8.5 \mathrm{~m} / \mathrm{s}$ the upstream boundary layer becomes turbulent as it can be deduced by the drastic change in the spectral structure of figure 3 at this velocity, and less sensitive to the acoustic forcing. One important feature emerging from this figure is that, for $u_{e}>11 \mathrm{~m} / \mathrm{s}$, a weak energy peak with a characteristic low frequency around $5 \div 10 \mathrm{~Hz}$, increasing with the speed, appears. As it will be shown later, this peak is due to a lowfrequency oscillatory motion of the cell flow.

Similar couplings between facility resonance and laminar shear flows are frequently encountered in experimental investigations, e.g. Chatellier et al (2004) for a cavity flow experiment. From a practical viewpoint, this phenomenon may be of paramount concern because a strong fluid-structure interaction between the cell flow and the structure hosting the cell might occur, Olsman et al (2011).

Two different conditions were chosen to be further investigated in detail. The first will be referred to as case $\mathrm{C} 1$ in the following and corresponds to $u_{e}=6 \mathrm{~m} / \mathrm{s}$, where the shear layer flow displays intense forced oscillations, resulting from the coupling between the facility resonance and the fourth cavity mode. In this condition, the upstream boundary layer is laminar and its momentum thickness $\theta_{0}$ is small with respect to the cavity length $L$. In the second condition, referred to as case $\mathrm{C} 2, u_{e}=12.40 \mathrm{~m} / \mathrm{s}$ and the shear layer does not develop self-sustained oscillations as the ratio between the opening length and the momentum thickness of the turbulent incoming boundary layer is well below the value at which self-sustained oscillations are expected For both cases the upstream boundary layer suction system is set at the maximum value. The rele- vant parameters of the two conditions are reported in table 1 . In the table, $\delta_{0}^{*}$ is the displacement thickness of the upstream boundary layer, measured at the reference station $x / L=-0.14 \mathrm{~mm}$, while $H_{0}=\delta_{0}^{*} / \theta_{0}$ is the shape factor.

\subsection{Results for case $\mathrm{C} 1$}

\subsubsection{Shear layer evolution}

Figure 4 displays the streamwise evolution of vertical profiles of the mean velocity $\bar{u}(x, y)$. For each profile, two dashed lines indicate $\bar{u}(y) / u_{e}=0$ and $\bar{u}(y) / u_{e}=1$. Furthermore, the line for $u=0$ indicates the position where each velocity profiles was measured. The geometry of the downstream part of the cell is also indicated.

A laminar shear layer develops from the upstream boundary layer detaching from the cusp and thickens as it develops downstream. In particular, the thickening occurs towards the interior of the cavity, because the topmost layers of fluid inside the cavity are entrained and accelerated by weak viscous momentum transport. The flow velocity inside the cell is on the order of 0.05 , a clear evidence of poor recirculation of the flow.

The mean velocity profiles also indicate that as the shear layer flow reaches the impingement region, on the downstream part of the cavity, it lifts up. As a result, the thickness of the boundary layer flow developing from this region is significantly larger than $\delta_{0}$ and it is comparable to that of the shear layer at the impingement.

The streamwise evolution of vertical profiles of the root-mean-square value of the normalised velocity fluctuations $u_{r m s}^{\prime} / u_{e}$ is shown in figure 5. The maximum value of $u_{r m s}^{\prime} / u_{e}$ across the shear layer is calculated for each profile and the evolution of this quantity is also plotted in the same figure, with red line and symbols, to provide the intensity scale for the profiles. Several regions can be identified.

The intensity of the fluctuations remains practically the same for the first third of the shear layer, up to about $x / L \approx 0.35$. In this first region, the value of $u_{r m s}^{\prime} / u_{e}$ is the same as in the upstream boundary layer, i.e. about $4.5 \%$. The profiles do not change significantly, even though spectral analysis of the velocity time histories at the peak location, not reported here, indicates that the fluctuation energy in a narrow band around the resonance frequency grows exponentially in this laminar shear flow with the streamwise coordinate, by about five orders of magnitude in the first half of the opening length. Before $x / L \approx 0.35$, this energy is small and lower than the broadband fluctuations in the upstream boundary, but past this point, the second re- 


\begin{tabular}{ccccccccccc}
\hline Case & $u_{e}[\mathrm{~m} / \mathrm{s}]$ & $R e_{L}$ & $\delta_{0}[\mathrm{~mm}]$ & $D / \delta_{0}$ & $\theta_{0}[\mathrm{~mm}]$ & $R e_{\theta_{0}}$ & $L / \theta_{0}$ & $\delta_{0}^{*}[\mathrm{~mm}]$ & $H_{0}$ & $C_{f}$ \\
\hline C1 & 6.0 & 27000 & 4.4 & 25.9 & 0.60 & 240 & 113 & 1.32 & 2.20 & $3.4 \times 10^{-7}$ \\
C2 & 12.4 & 56000 & 22.0 & 5.2 & 2.05 & 9000 & 34 & 2.96 & 1.44 & $3.95 \times 10^{-3}$ \\
\hline
\end{tabular}

Table 1 Values of the parameters for the two cases discussed in this paper. The skin friction coefficient $C_{f}$ of the upstream boundary layer is estimated from a least-squares fit of the Blasius solution, for case C1, and with Clauser method, for case C2.

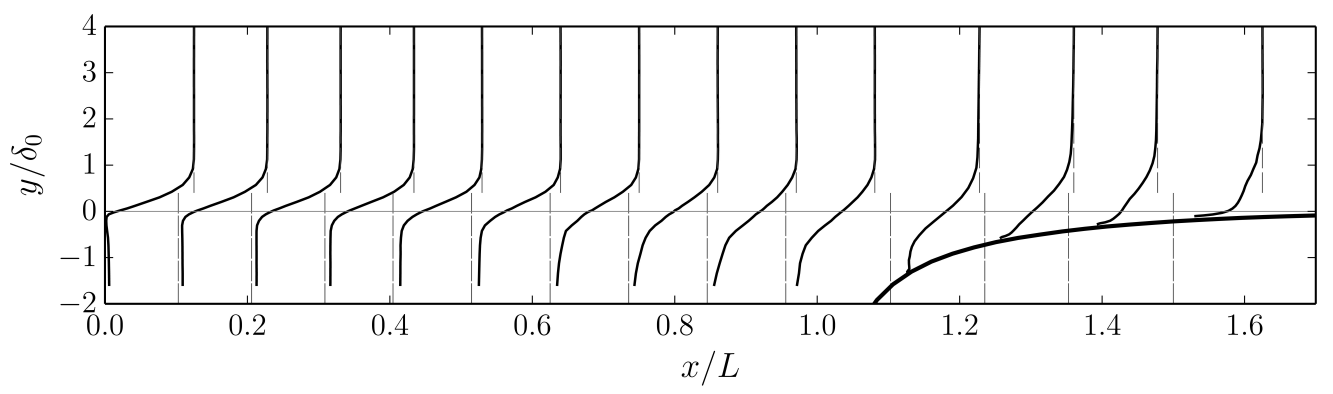

Fig. 4 Selected profiles of $\bar{u}(y)$ along the shear layer for case C1. The horizontal and vertical axes are in scale.

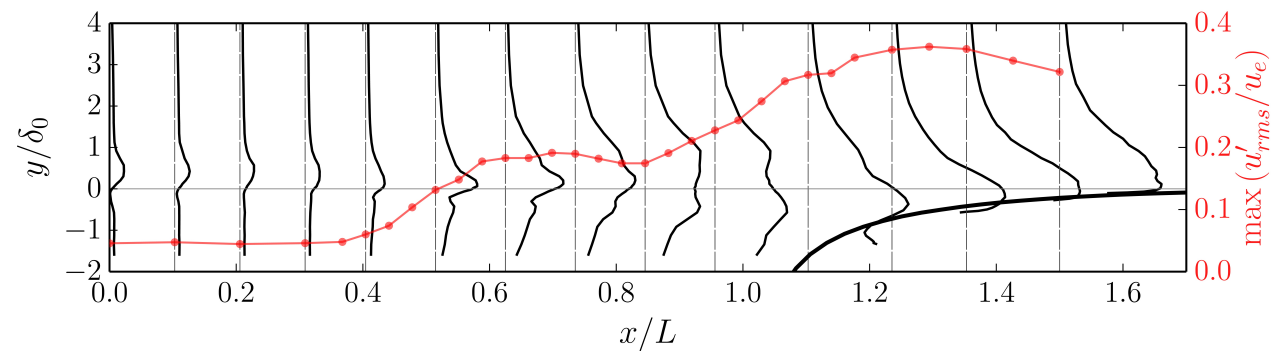

Fig. 5 Selected profiles of $u_{r m s}^{\prime} / u_{e}$ along the shear layer. The red curve is the streamwise evolution of $\max \left(u_{r m s}^{\prime} / u_{e}\right)$. The horizontal and left vertical axes are in scale.

gion, the amplitude of the Kelvin-Helmoltz instability mode dominates the fluctuations in the shear layer. At around $x / L \approx 0.6$, nonlinear saturation of this linear amplification mechanism occurs and the fluctuation energy reaches a constant value, about 0.18 , up to around $x / L \approx 0.85$. A small part of the velocity time history at $y=0$ for this location is reported in figure 6 , (black solid line), showing a very coherent oscillatory behaviour. The vertical area interested by the fluctuations increases remarkably. This means that the $y$-spatially-averaged value of $u_{r m s}^{\prime} / u_{e}$ increases more than $\max \left(u_{r m s}^{\prime} / u_{e}\right)$. A second peak, common for cavity flows, e.g. Basley et al (2010), appears on the profile in this region, at around $y / \delta_{0}=-0.45$. This peak becomes dominant after $x / L \approx 1$ and affects the near wall region when the flow reattaches.

The fluctuation energy increases further in a third region, after $x / L \approx 0.85$, due to the turbulent break up of the coherent rollers of spanwise vorticity developing in the shear layer, as they approach the impingement region. Evidence of this phenomenon will be given by the flow visualisations reported in section 3.2.4, although

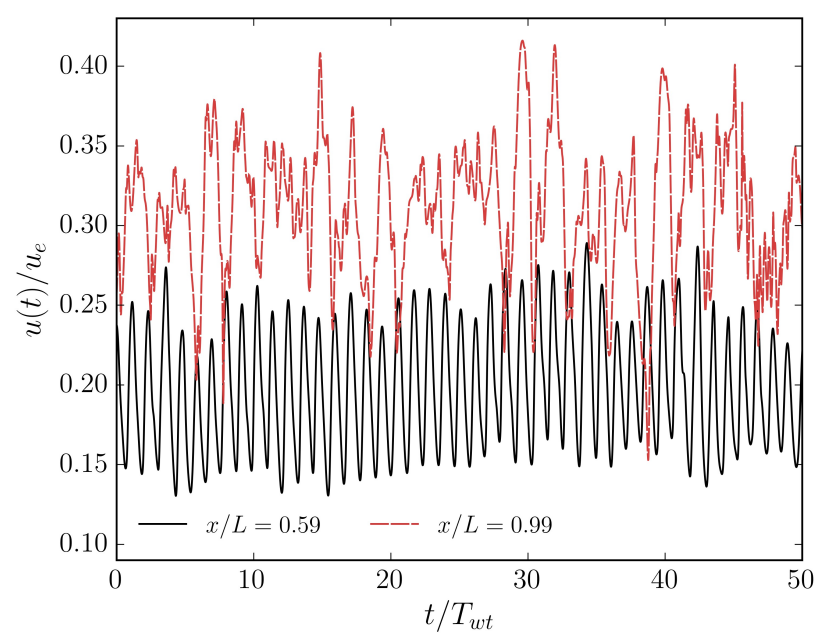

Fig. 6 Velocity time histories in two characteristics locations in the shear layer at $y=0$. Time is normalised with the period $T_{w t}$ of the facility resonance.

the time history reported in figure 6 for $x / L=0.99$ clearly shows how the coherent motion in the first part of the shear layer is disrupted and turbulent behaviour 
appears. The increase continues past the end of the cavity, up to $x / L \approx 1.3$, where the intense velocity fluctuations are due to the turbulent wall flow developing in this region. The overall behaviour is a strong increase of the velocity fluctuations all along the shear layer, resulting in a highly turbulent flow reattaching on the downstream part of the cell and developing downstream from there.

\subsubsection{Spectral and correlation analyses}

Panels $(a),(b)$ and $(c)$ of figure 7 show the power spectral density of the normalised velocity fluctuations at three significant points in the cell. The points are also indicated in panel $(d)$ of the same figure, which displays the spatial distribution of the spectral density at the fundamental frequency $f_{w t}$. The value is obtained by integration of the spectral density in a frequency band wide $1 \mathrm{~Hz}$ around $f_{w t}$, for each point in the shear layer where a velocity time history was measured.

Point $(a)$ refers to $x / L=0, y / \delta_{0}=-3.4(y / L=$ $-0.22)$, inside the cell and under the cusp. Point $(b)$ refers to $x / L=0.58, y / \delta_{0}=0.45$, in the most unsteady region of the shear layer. Point (c) refers to $x / L=1.0$, $y / \delta_{0}=-0.45$, near the shear layer impingement region on the downstream part of the cell. In the plots, the frequency is reported in dimensional units because the entire flow is locked onto the resonance and it would not be appropriate to report it in terms of Strouhal number.

The three spectra in the top panels are characterised by a sharp peak at $f_{w t}$. However, in the cell region, figure $7-(a)$ the spectrum is dominated mostly by low frequencies components due to slow dynamics of the recirculating flow, whereas energy at $f_{w t}$ is relatively small. In the shear layer, figure $7-(b)$, the velocity fluctuations at the fundamental frequency dominate the spectrum. Several other peaks of decreasing intensity at the higher harmonics of $f_{w t}$ can be detected. In the shear layer impingement region, figure 7-(c), the spectrum is still dominated by the fundamental frequency and its first harmonic, but shows characteristics of a turbulent flow. This is a demonstration of the fact that the coherent spanwise vorticity rollers being advected in the shear layer break up in this region, resulting in a highly turbulent flow developing downstream, although the signature of the shear layer oscillations is still clear.

The spatial distributions of the fluctuation energy at the fundamental frequency, displayed in figure $7-d$, illustrates that the entire external incoming flow is permeated by weak velocity fluctuations at the fundamental frequency. This fluctuation forces the initial region of the shear layer which then develops very intense fluc- tuations. The exponential amplification of the fluctuation energy in the first part is visible, especially in the region for $0<y / \delta_{0}<0.5$. As it was discussed for the profiles of $u_{r m s}^{\prime} / u_{e}$, the fluctuation amplitude eventually saturates at around $x / L=0.6$, followed by a significant vertical spreading of the region where intense fluctuations are observed, characterised by two lobes, one around $y / \delta_{0} \approx 1$, the other around $y / \delta_{0} \approx-0.5$.

These results are in good qualitative agreement with those obtained for laminar shear layers bounding rectangular cavities. For example, Basley et al (2010), investigated a rectangular cavity flow characterised by $R e_{L}=12000$, about half of that of the current experiment, with a ratio $L / \theta_{0}=77$, thus with a laminar boundary layer slightly thicker than that of the present case. These authors reported an energy distribution at the first shear layer mode frequency which presented the same qualitative features as those in figure 7-d. Such a similarity essentially indicates that the basic mechanism at the hearth of the phenomenon is the same, despite the different geometry.

Further insight can be gained by spectral density analysis of the normalised pressure fluctuations $p_{j}^{\prime} / q_{e}$, reported in figure 8 for the three microphones. The two most energetic features of the spectrum of the signal $p_{1}^{\prime}$, from the microphone located inside the cell under the cusp, figure $8-(a)$ are the sharp peaks at $f=f_{w t}$ and $2 f_{w t}$. The spectrum also shows a large number of other peaks in the range $200 \mathrm{~Hz}<f<1000 \mathrm{~Hz}$. These have been associated almost entirely to coloured noise generated by the experimental setup. Nonetheless, the dynamics of the cavity flow occurring in this band are quite weak, due to the low velocity of the fluid in the cell, such that their signature is entirely covered by background noise sources. Interestingly, there is no clear evidence of energy peaks at the harmonics of $f_{w t}$ higher than the second. This is in stark contrast with the spectrum of the velocity signal in the shear layer, see figure $7-(b)$, where harmonics up to at least the sixth were clearly identifiable.

The signal $p_{2}^{\prime}$ shows quite high energy levels across the entire spectrum. This behaviour is representative of the intense turbulent pressure fluctuations in the shear layer impingement region. By contrast the spectrum of signal $p_{3}^{\prime}$, panel $c$, obtained from the microphone located downstream of the cavity, has quite small energy at the fundamental frequency. This difference clearly indicates how the cavity flow is subject to a strong pressure-velocity coupling that dramatically increases the intensity of the pressure fluctuations.

In figure 9 the cross-correlation function between the velocity fluctuations at $x / L=0.58, y / \delta_{0}=0.45$, in the most unsteady region of the shear layer, (as in panel 

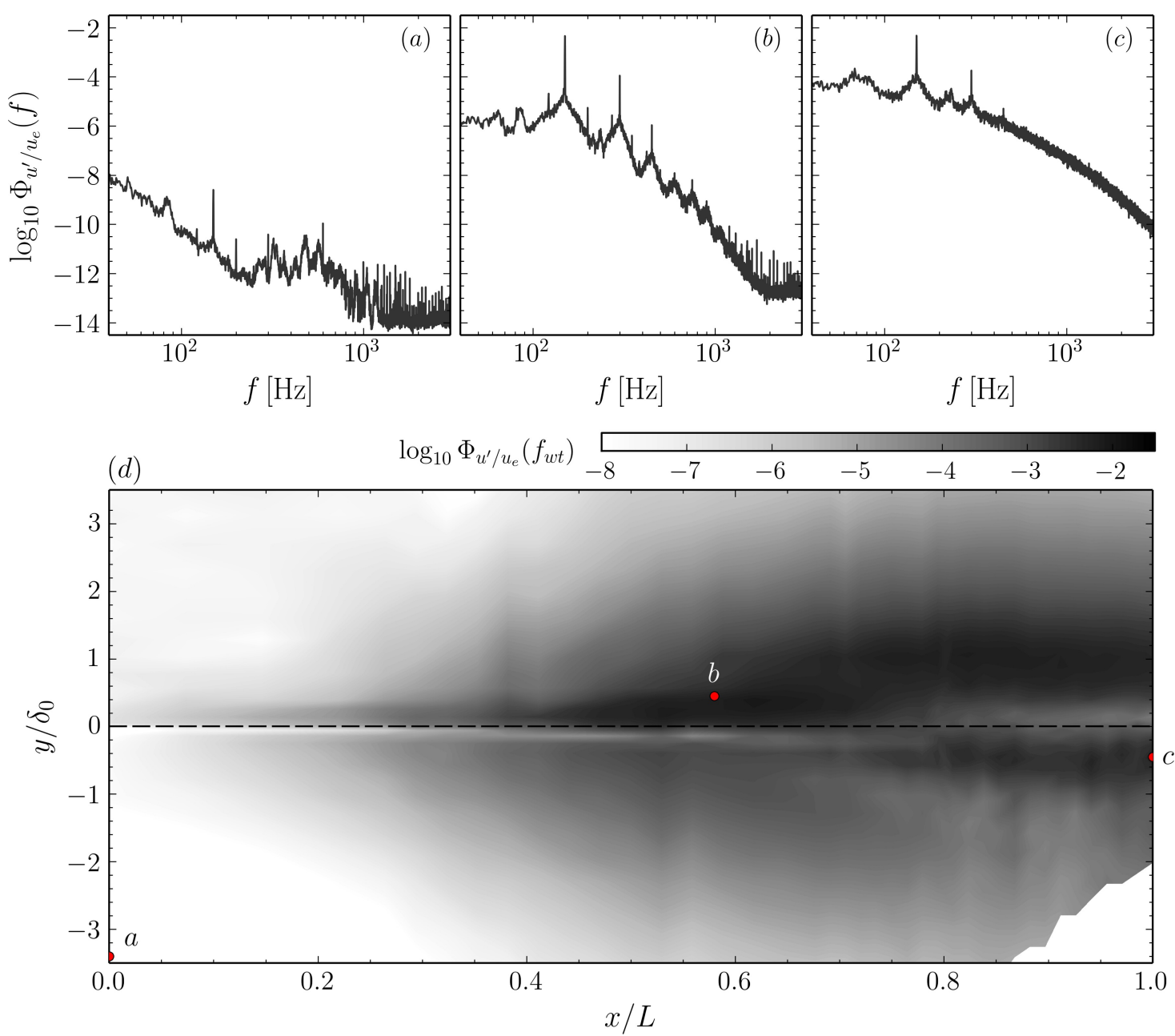

Fig. 7 Power spectral densities of the normalised velocity fluctuations $u^{\prime} / u_{e}$, at three reference points: $(a)-x / L=0, y / \delta_{0}-3.4$; (b) $-x / L=0.58, y / \delta_{0}=0.45 ;(c)-x / L=1.0, y / \delta_{0}=-0.45$. The locations of these points is indicated in panel $(d)$, which also shows the spatial distribution of the power spectral density of the normalised velocity fluctuations in a narrow band around the fundamental frequency.
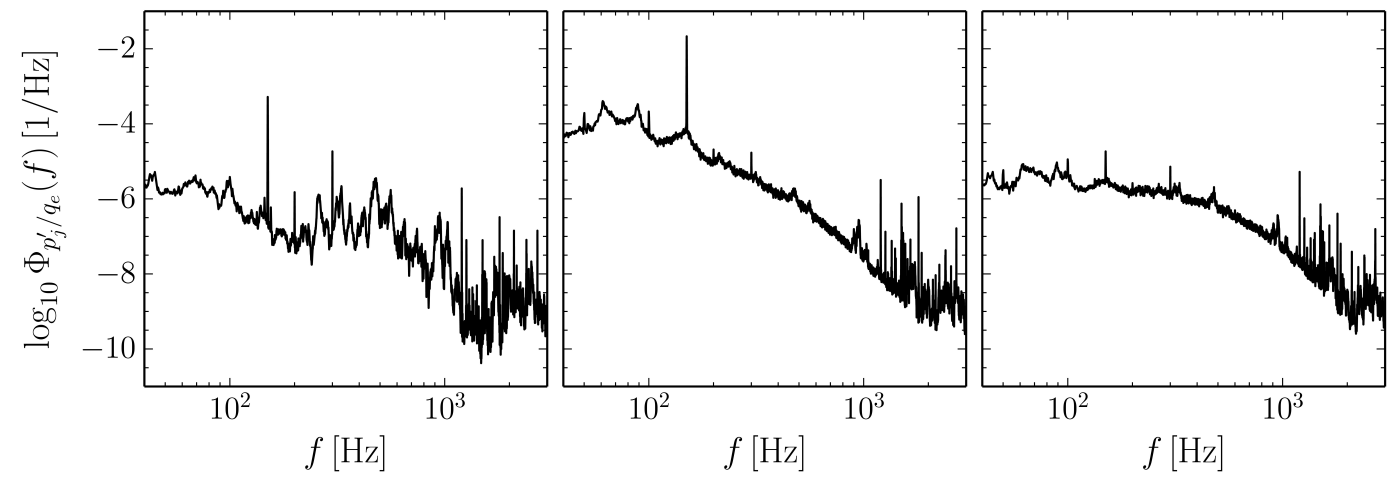

Fig. 8 Power spectral densities of the normalised pressure fluctuations $p_{j}^{\prime} / q_{e}$, for signal $p_{1}^{\prime},(a)$, signal $p_{2}^{\prime},(b)$ and $p_{3}^{\prime}$, (c), (see figure 1 for the locations). 
$b$ of figure 7 ), and the pressure fluctuations $p_{1}^{\prime}, p_{2}^{\prime}$ and $p_{3}^{\prime}$, is reported in panels $(a),(b)$ and $(c)$, respectively. The cross-correlation function is defined as

$\rho_{u^{\prime} p_{j}^{\prime}}(\tau)=\frac{\overline{u^{\prime}(t) p_{j}^{\prime}(t+\tau)}}{{\overline{u^{\prime}(t)^{2}}}^{1 / 2}{\overline{p_{j}^{\prime}(t)^{2}}}^{1 / 2}}$.

The cross-correlation function for signals $p_{1}^{\prime}$ and $p_{2}^{\prime}$ highlights the strong pressure-velocity coupling occurring in the cell. It also shows the periodic nature of the fluctuations, with period approximately equal to the inverse of $f_{w t}$. The correlation level is significant and on the order of $0.4 \div 0.6$, for $p_{1}^{\prime}$ and $p_{2}^{\prime}$. By contrast, the correlation level with $p_{3}^{\prime}$ is lower and no clear periodicity can be identified. This can be explained by considering that microphone $m 3$ is embedded in the wall downstream of the cavity, where a completely different flow develops.

The level of correlation between the wall-pressure and the velocity fluctuations is function of the point where the velocity is measured. The cross-correlation coefficient function was calculated for each point in the shear layer where a velocity time series was sampled, and for lags up to 750 periods of the main oscillation. Then, the maximum over $\tau$ of the absolute value of the cross-correlation is extracted as an indicator of the correlation level of the wall-pressure fluctuations measured from microphone $m_{j}$ with the velocity fluctuations at each point.

Figure 10 shows the results of this analysis for signal $p_{1}^{\prime}$, located under the cusp of the cell. The colour map shows some irregularity, mostly due to the limited spatial resolution of the hot wire measurements and to the limited acquisition times. Nonetheless, the results clearly indicates where the velocity fluctuations are more correlated with the wall-pressure fluctuations. This occurs in two horizontally elongated lobes, symmetrically located above and below $y / \delta_{0}=0$, developing from $x / L \approx 0.3$, with the upper one extending far downstream $x / L=1$. The correlation value in these lobes is high, on the order of 0.4-0.6. Although not shown here, the correlation map constructed using signal $p_{2}^{\prime}$ is analogous to that of figure 10 , and shows similar correlation levels and a similar spatial distribution. On the other hand, the correlation of the velocity fluctuations using signal $p_{3}^{\prime}$ is weaker, across the entire flow field.

The correlation map shown in figure 10 is remarkably similar to the spectral density map at $f_{w t}$ reported in figure $7-(d)$, because most of the correlation is due to the fluctuations of the shear layer at $f_{w t}$. This can be noticed from the squared coherence function between the wall-pressure and the velocity fluctuations, defined as

$$
\gamma_{u^{\prime} p_{j}^{\prime}}^{2}(f)=\frac{\left|\Phi_{u^{\prime} p_{j}^{\prime}}(f)\right|^{2}}{\Phi_{u^{\prime}}(f) \Phi_{p_{j}^{\prime}}(f)}
$$

where $\Phi_{p_{j}^{\prime}}$ and $\Phi_{u^{\prime}}$ are the power spectral densities of the $j$-th wall-pressure signal and of the velocity signal, respectively, and $\Phi_{u^{\prime} p_{j}^{\prime}}(f)$ is the cross spectral density between the velocity and wall-pressure fluctuations. An example of the extensive analyses which have been conducted in this direction is given in figure 11, which reports the squared coherence function for velocity fluctuations measured at $x / L=0.58, y / \delta_{0}=0.45$. The pressure-velocity coupling is very narrow-band in nature, since the coherence function is statistically different from zero only at few distinct frequencies. At $f_{w t}$ the squared coherence function for the two wallpressure signals measured inside the cell can be as high as 0.95 . However, for signal $p_{3}^{\prime}$, panel $(c)$, the coherence at $f_{w t}$ is quite small, as it was expected by observation of the cross-correlation functions. At the first harmonic, the coherence function is about 0.75 for signal $p_{1}^{\prime}$, and is as low as 0.25 for signal $p_{2}^{\prime}$. Interestingly, the coherence at the higher harmonics is practically zero.

The coherence function is characterised by several other peaks at frequencies not equal to that of the harmonics. However, despite the relatively high levels of coherence these peaks seem to have little dynamic relevance for the cell flow, as the energy of the velocity fluctuations in the top panels in figure 7 is quite small.

\subsubsection{Effects on boundary layer}

The boundary layers upstream and immediately downstream of the cell are now compared and results are discussed in the light of the flow features presented above.

Figure 12- $(a)$ displays the mean velocity profiles of the boundary layer upstream, $x / L=-0.14$, and downstream, $x / L=2.05$, of the cell. The boundary layer downstream of the cell is fully turbulent as a result of the strong amplification of the velocity fluctuations in the shear layer, and the subsequent break up of the coherent vorticity rollers. The intense turbulent fluctuations arising from this interaction, figure 12-(b), extend far in the outer flow and large values of $u_{r m s}^{\prime} / u_{e}$ can be found up to $3 \delta_{0}$. The mean velocity profile at $x / L=2.05$ is characterised by higher momentum in the near wall region with respect to the upstream boundary layer, even though there is a consistent velocity deficit with respect to upstream from $y / \delta_{0} \approx 0.6$ upwards.

It is argued that the net effects of a TVC on the aerodynamic performance of a TVC-controlled airfoil are indirectly related to the variation across the cell of 


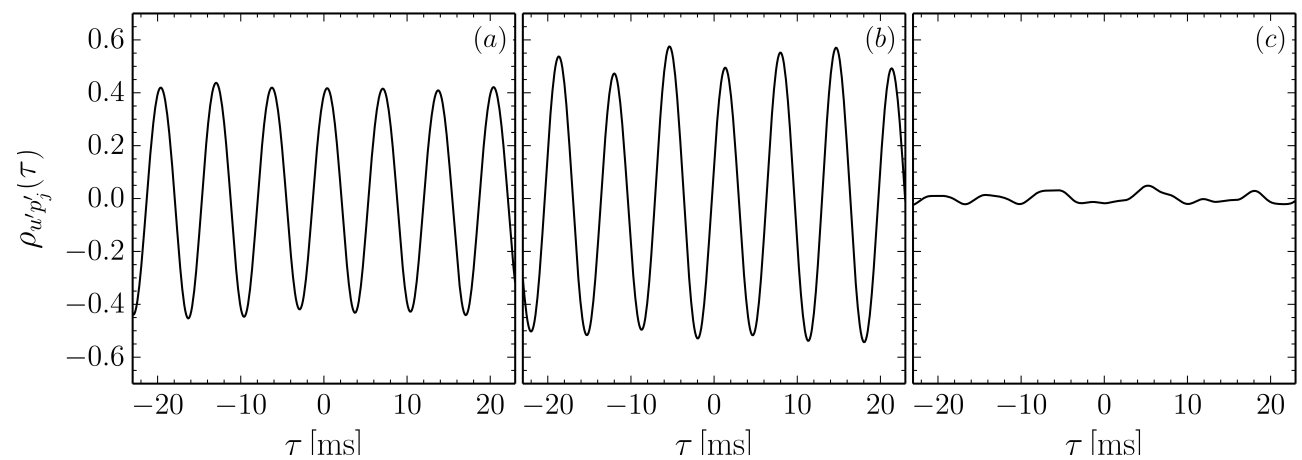

Fig. 9 Cross-correlation functions $\rho_{u^{\prime} p_{j}^{\prime}}(\tau)$ for velocity fluctuations measured at $x / L=0.58, y / \delta_{0}=0.45$ and wall-pressure signals $p_{1}^{\prime}, p_{2}^{\prime}$ and $p_{3}^{\prime}$, in panels $(a),(b)$ and $(c)$, respectively.

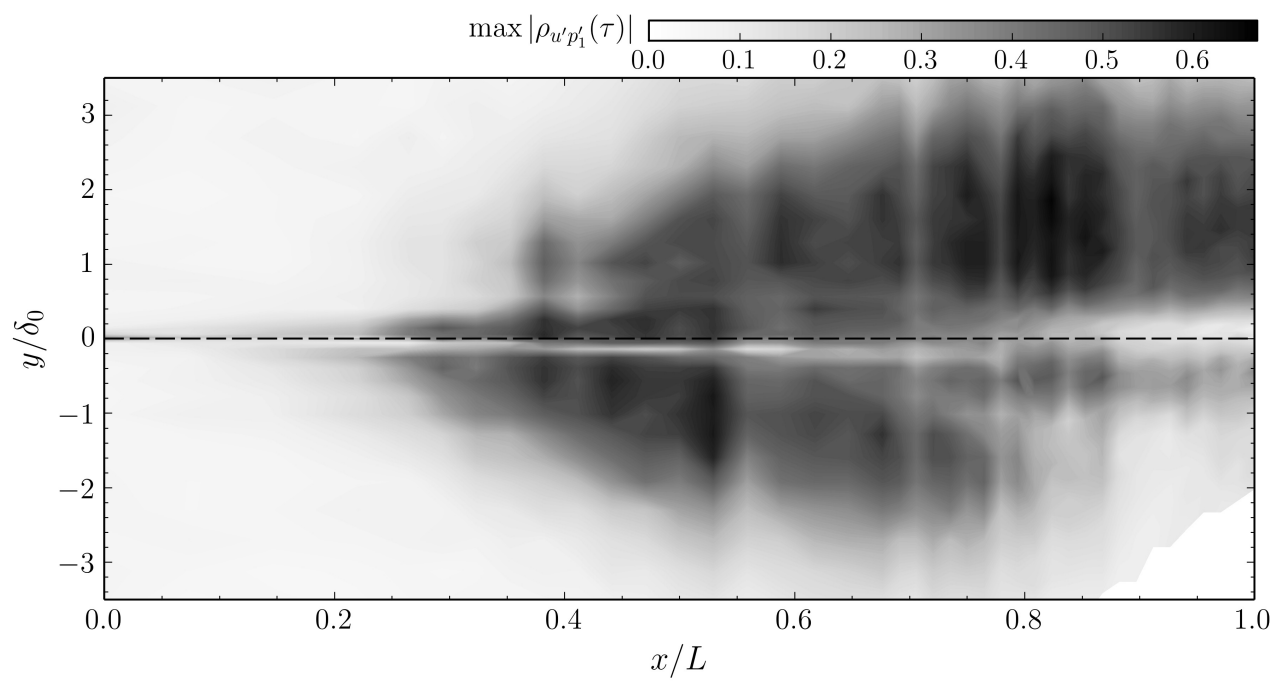

Fig. 10 Colour map of the maximum absolute value of the cross-correlation coefficient functions $\rho_{u^{\prime} p_{1}^{\prime}}$, across the entire shear layer.

the boundary layer momentum thickness. A strong localised increase of this quantity, larger than what would naturally occur as a result of skin friction on a solid wall, might result in a higher drag coefficient. This hypothesis might apply in particular at low angles of attack, when the boundary layer on the airfoil is fully attached and skin friction drag is the dominant contribution. In the present case, the momentum thickness increased from $0.60 \mathrm{~mm}$ to $1.4 \mathrm{~mm}$, indicating a consistent loss associated to the formation, exponential amplification and break up of coherent vorticity rollers in the shear layer. It is worth pointing out that the occurrence of Kelvin-Helmoltz instabilities depends on the ratio between the incoming boundary layer momentum thickness and the cavity opening, Gharib and Roshko (1987), and occurs for both turbulent and laminar shear layers, see e.g. Rockwell and Naudascher (1978); Lawson and Barakos (2011). This large positive variation might help explaining the large drag coefficient of TVCcontrolled airfoils in previous experiments at low angles of attack, e.g. De Gregorio and Fraioli (2008); Lasagna et al (2011), when no active strategy to control the flow in the cell itself was applied.

\subsubsection{Flow visualisations}

In figure 13 a sequence of six consecutive snapshots of a smoke flow visualisation for case $\mathrm{C} 1$, taken in the mid-span section of the cell, is reported. The snapshots were sampled at $100 \mathrm{~Hz}$, hence the shear layer oscillations are under-sampled, and the vorticity dynamics in this region cannot be distinguished. However, the low sampling frequency allowed a higher exposure time $(9997 \mu \mathrm{s})$ which enabled a better observation of the dynamics of the internal cell flow, where hot-wire measurements would have been too intrusive. The upstream part of the cavity flow is not clearly visible, due to the shadow cast by the cell cusp. The images were post-processed by subtraction of the time averaged image and by black/white equalisation to better 


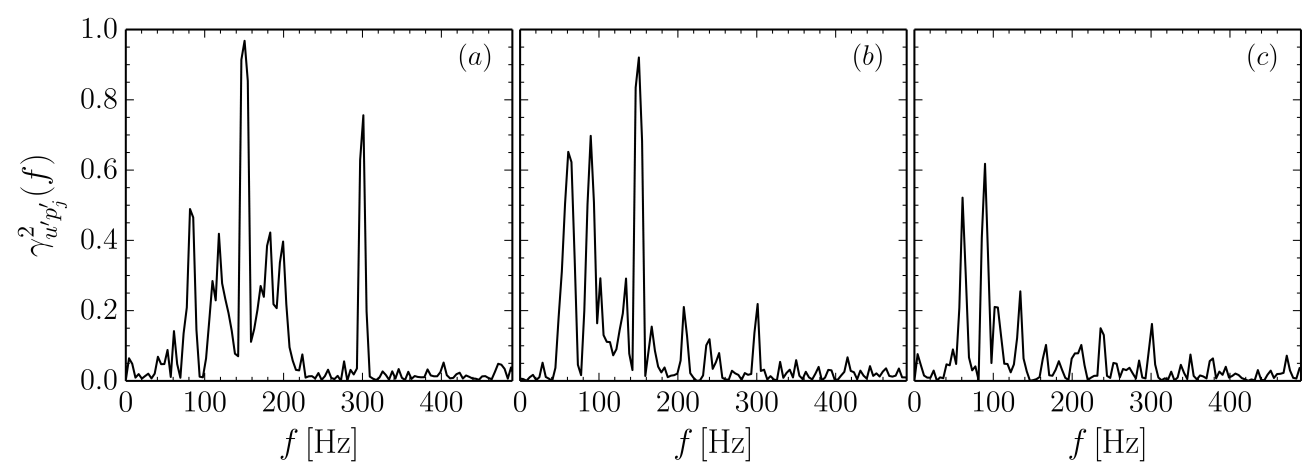

Fig. 11 Squared coherence functions $\gamma_{u^{\prime} p_{j}^{\prime}}^{2}$ for velocity fluctuations measured at $x / L=0.58, y / \delta_{0}=0.45$ and wall-pressure signals $p_{1}^{\prime}, p_{2}^{\prime}$ and $p_{3}^{\prime}$, in panels $(a),(b)$ and $(c)$, respectively.
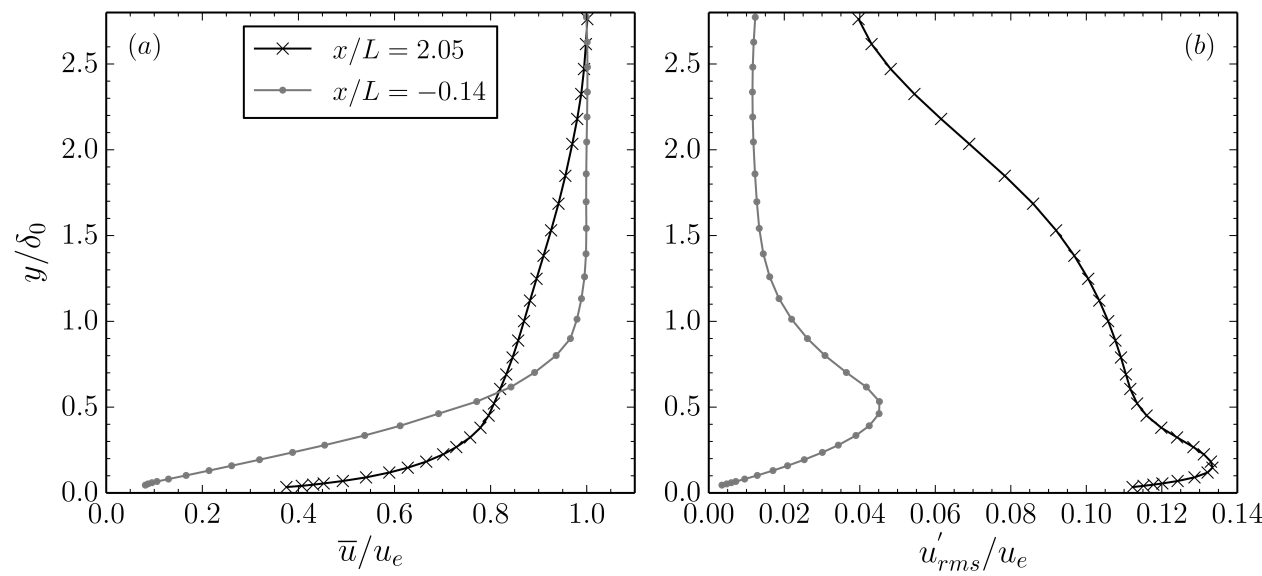

Fig. 12 Comparison of upstream $x / L=-0.14$, and downstream, $x / L=2.05$, boundary layer profiles. Mean velocity, (a), and root-mean-square value of velocity fluctuations, $(b)$.

highlight significant flow structures in the cell region. One every two sampled snapshots is reported, separated by $\Delta t=2 / 100 \mathrm{~s}$. Note that the time required for the external flow to cover the cavity opening length is $L / u_{e}=0.0113 \mathrm{~s}$. The sequence is sorted left-right, top-bottom, and a movie is available online as supplementary material.

The snapshots show the presence of an irregular recirculating vortical flow, with a centre slightly shifted downstream with respect to the centre and towards the cell opening, as also observed in Donelli et al (2009a) and Baranov et al (2000). Furthermore, a wide range of smaller structures advected by the main rotation can be observed especially in the downstream region of the cell.

The visualisations suggest possible mechanisms driving the rotation of the cell flow. A first contribution is the viscous momentum transfer from the external flow through the shear layer, which only accelerates the uppermost layers. However, it is argued that this mechanisms alone cannot explain the observed rate of rotation. A further contribution is the intermittent injection of fresh, high momentum fluid packets from the external flow downwards in the downstream region of the cell. The motion of one of these packets can be observed in the snapshots as a large black area, hence external fluid with no tracer, flowing from the downstream part of the cell along the internal wall. The injection of these packets is highly erratic as does not appear to occur as a particular frequency.

An analogous mechanism was observed by Hokpunna and Manhart (2007) in a large eddy simulation of a similar configuration, although at a higher Reynolds number and with a turbulent incoming boundary layer. A similar phenomenon has been also observed for rectangular cavities, although geometry of the impingement region is sharp and not rounded as in the present case. For example Faure et al (2006) evidenced a periodic injection of mass into the cavity connected to the shear layer oscillations resulting in a pulsed jet-like flow.

The flow visualisation does not show large-scale internal flow separation, as the vortex core fills completely the cavity and the internal boundary layer is fully attached over the entire wall. This is in contrast to the complex internal flow topology observed in rectangular open cavity flows, e.g. Faure et al (2006); Faure (2014), 

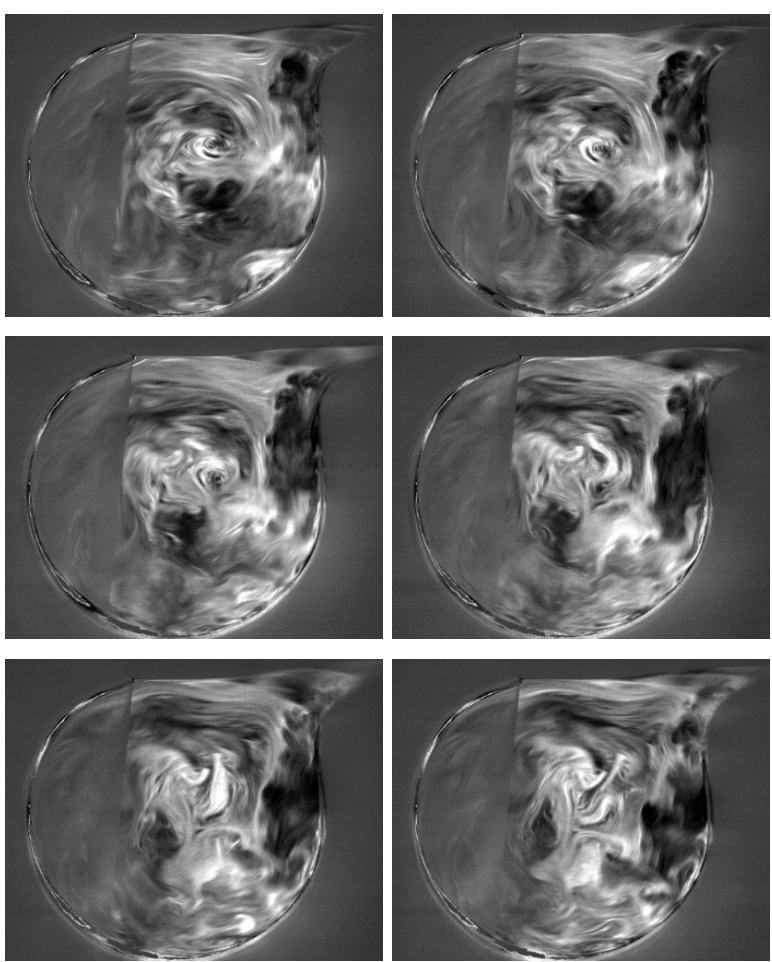

Fig. 13 Sequence of smoke flow visualisation snapshots for case $\mathrm{C} 1$, sorted left to right, top to bottom. Time difference between snapshots is $2 / 100 \mathrm{~s}$.

where secondary separation is a relevant feature, and affects remarkably the shear layer dynamics, Kuo and Huang (2001). Rigorous results, Bunyakin et al (1998), show that a region of the wall with adverse pressure gradient has to exist in a vortex cell. For this geometry, this location is in the downstream part of the cell. Soon after the flow impinges on the downstream region, it accellerates backwards, bends around the rounded corner, and then encounters a short region of adverse pressure gradient, see figure 19 in Donelli et al (2009a). In the present case, the intense injection of turbulent flow from the impingement region down into the cavity seems to prevent large separation in this region. However, small regions of reverse flow can be occasionally observed in the flow visualisation movie in the downstream internal part of the cell, although these are highly transient and have a relatively small size.

\subsection{Three-dimensional properties of the flow}

The three dimensional character of the flow has been investigated by traversing the hot wire probe along the $z$ axis at several fixed $x, y$ positions.

As a representative example, figure 14 shows profiles of $\bar{u}(z) / u_{e}$, in panel $(a)$, and $u_{r m s}^{\prime}(z) / u_{e}$, panel (b), measured at $x / L=0.59, y / \delta_{0}=-1.69$, inside the
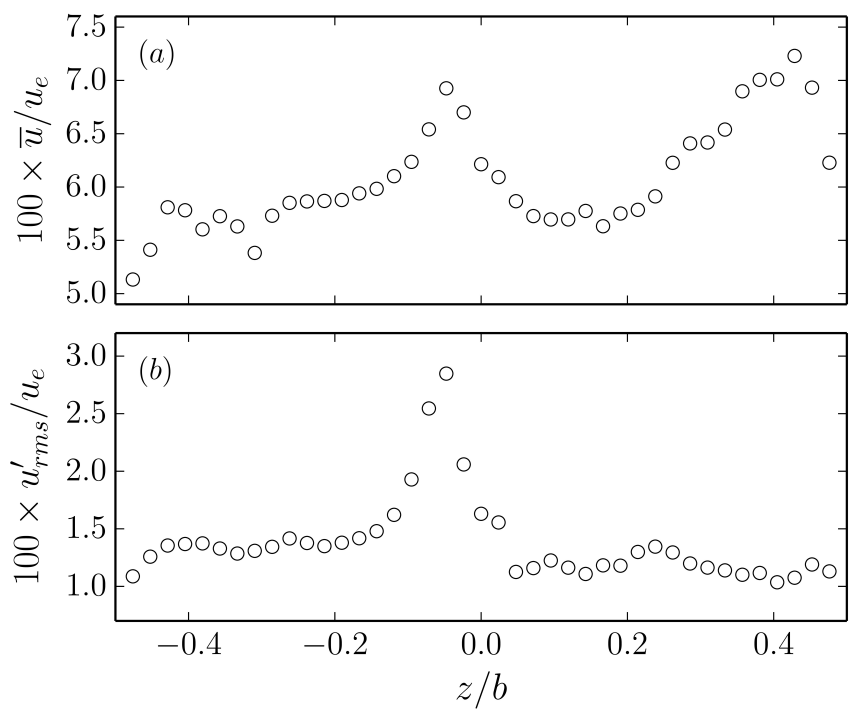

Fig. 14 Spanwise profiles of mean velocity, $(a)$, and rootmean-square value of the velocity fluctuations, $(b)$, measured at $x / L=0.59, y / \delta_{0}=-1.69$, inside the cavity.

cavity region. The spanwise coordinate is made nondimensional with the cavity width. The most striking feature is observed in the behaviour of the root-meansquare value of the velocity fluctuations. The data are characterised by a sharp peak slightly on the left of the mid-span section. The value of $u_{r m s}^{\prime}(z) / u_{e}$ is more than twice the average value found along the span at that $x, y$ position. Furthermore, the flow is clearly not symmetric with respect to the mid-span as a drift in the mean velocity is also observed, for positive $z / b$.

A similar asymmetry of the flow was also observed in numerical simulations of compressible flows over a rectangular cavity by Larchevêque et al (2007). These authors suggested that such behaviour is connected to an odd constraint of the natural wavelength of the threedimensional modulation by a finite span of the cavity. Three dimensional characteristics in rectangular cavity flows have been observed by several researchers in both numerical and experimental setups, e.g. Maull and East (1963); Larchevêque et al (2007); Faure et al (2006); Brès and Colonius (2008); de Vicente et al (2014) for open cavities and by Kuhlmann et al (1997) in liddriven configurations. Evidence of 3D modulations of the flow in trapped vortex cells has also been pointed in several investigations under VortexCell2050 project, both by numerical simulations, e.g. Hokpunna and Manhart (2007), as well as in experiments, Savelsberg and Castro (2008); Lasagna et al (2011).

As discussed in Savelsberg and Castro (2008), a Görtler type linear instability mostly observed in lowReynolds number cavity flow experiments, Faure et al (2006), arising in the present in the boundary layer 


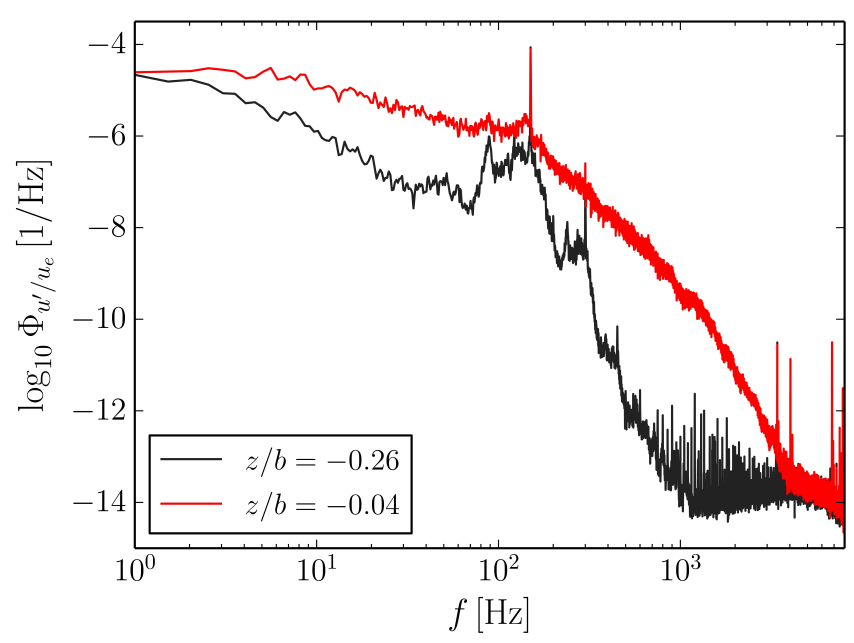

Fig. 15 Spectra of the velocity fluctuations at $z / b=-0.04$ and $z / b=-0.26, x / L=0.59, y / \delta_{0}=-1.69$

flowing along the concave cell wall would have a wavelength on the order of the boundary layer thickness. However, the spanwise profiles of figure 14 show a quite long wavelength, much larger than a possible estimate of the internal boundary layer thickness from the mean velocity profiles of figure 4 . Hence, the modulation observed is probably not driven by this mechanism, but rather from an Ekman pumping mechanism driven by the end walls, producing two asymmetric flow cells in the spanwise direction.

Figure 15 shows power spectral density of the velocity fluctuations at the point of largest $u_{r m s}^{\prime}(z) / u_{e}$, $z / b=-0.04$, in red, and at one location of the left, $z / b=-0.26$, in black, characteristic of the background value. In the frequency domain, the peak of fluctuations in the mid-span region is associated to a larger energy over a broad range of frequencies, characteristic of a turbulent flow, even though the most significant feature of the spectrum is still the fundamental resonance frequency of the shear layer, at $f=150 \mathrm{~Hz}$. On the other hand at $z / b=-0.26$, the spectrum is significantly different and energy is concentrated in a narrow band between about 80 and $200 \mathrm{~Hz}$, where most of the dynamics of the cavity flow take place. This spectrum is indeed characterised by a sharp energy drop-off at about $300-400 \mathrm{~Hz}$, plateauing after $1 \mathrm{kHz}$ at the level of the background noise. Note that the energy at $f_{w t}$ is similar for the two spectra, hence the variation in rms value observed in figure 14 is due to energy off this peak.

A further investigation was performed to quantify the influence of such three-dimensionality of the cavity flow on the properties of the boundary layer downstream of it, which is of interest in flow control applications. Figure 16 shows results of this investigation at
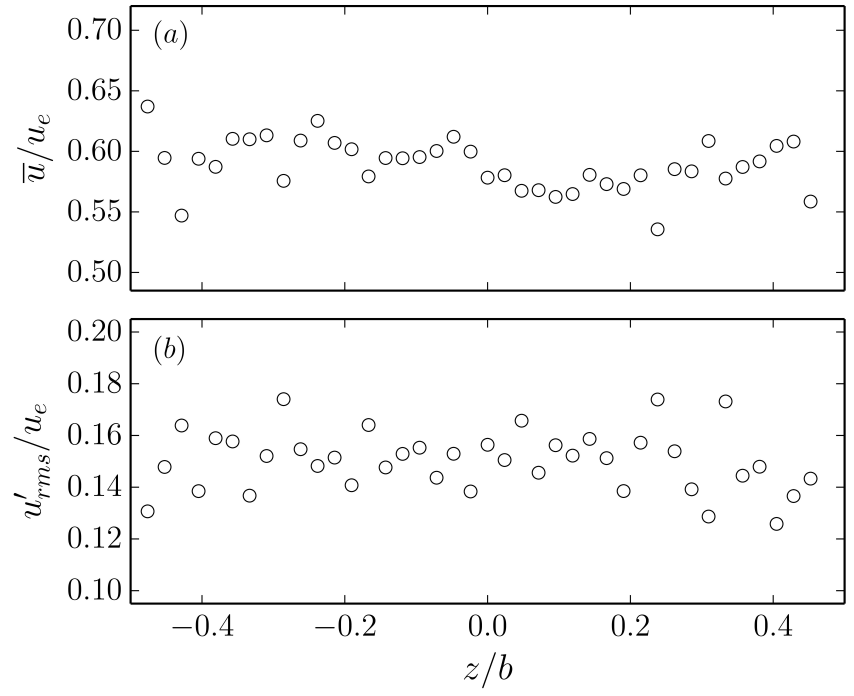

Fig. 16 Spanwise profiles of mean velocity, $(a)$, and of the root-mean-square value of the velocity fluctuations, $(b)$, measured at $x / L=1.32, y / \delta_{0}=0.00$, in the turbulent boundary layer downstream of the cavity.

$x / L=1.32, y / \delta_{0}=0.00$, further downstream of the cavity, where the wall flow is turbulent. Note that, although $y / \delta_{0}=0.00$, the hot wire probe is detached from the wall, due to the curvature of the cavity geometry. The top panel shows the profile of $\bar{u}(z) / u_{e}$, while the bottom one shows the transverse profile of $u_{r m s}^{\prime}(z) / u_{e}$. Downstream of the cavity, the flow has quite uniform behaviour in the spanwise direction, both in terms of mean velocity and rms, with no spanwise drifts, except for a moderate scatter around the space-averaged value mostly due to the limited sampling time. The absence of a strong three-dimensional structure in the wall-bounded flow downstream of the cell suggests a weak interaction between the three-dimensional modulation of the internal cavity flow and the evolution of the shear layer. This might suggests in turn that the threedimensional structure of the flow in the cell might not directly affect the effectiveness of a TVC as a control device on airfoils.

\subsection{Results for case C2}

Case C2 is characterised by a turbulent incoming boundary layer and a higher Reynolds number. In this condition the shear layer develops from a boundary layer whose ratio $L / \theta_{0}$ is 34 , too small for this flow to exhibit large coherent fluctuations. This flow condition is interesting because the trapped vortex cell was meant to control flow separation on airfoils at high Reynolds number, e.g. 10-20 time larger than what reported here, with turbulent incoming boundary layers. In fact, the 
"moving wall" effect introduced in Donelli et al (2009a) is more effective for a turbulent boundary layer due to the higher skin friction coefficient and the larger loss of momentum in the near wall flow, per unit streamwise length with respect to the laminar case. The parameters of this flow condition are as close as the experimental setup allowed to the large eddy simulation performed by Hokpunna and Manhart (2007) on a similar configuration. In their work, the cavity Reynolds number $R e_{D}$ is equal to $9.6 \cdot 10^{4}$ and the ratio $\delta_{0} / D$ is equal to 0.25 .

\subsubsection{Shear layer evolution}

Figure 17 shows mean velocity profiles at selected streamwise locations along the shear layer, as in figure 4 . The first profile, at $x / L=0$, shows the signature of the cell cusp, dividing the incoming boundary layer from the cell flow. The value of the velocity associated to the outer region of the rotating cell flow is about 0.22 and thus the flow rotates faster in case $\mathrm{C} 2$ than in case $\mathrm{C} 1$. This is because the momentum transfer from the external high momentum fluid to the cell flow operated by the turbulent stresses through the shear layer is more effective in driving the rotation of the cell flow than the viscous transfer mechanism.

The first profile indicates the presence of a boundary layer flowing along the cell surface, induced by the rotating vortex core. The outermost layers of fluid in the cavity have a velocity close to that obtained by Hokpunna and Manhart (2007) and by Donelli et al (2009a) at the corresponding location. The velocity deficit associated with the cusp is quickly eroded and the thickness of the turbulent shear layer increases rapidly due to the high mixing operated by the turbulent fluctuations, spreading towards the interior of the cavity. Perhaps, the most salient feature is that when the flow reattaches, between $x / L=1$ and 1.2 , the mean velocity profile is characterised by a significant velocity deficit.

Figure 18 shows profiles of the normalised root-meansquare value of the velocity fluctuations $u_{r m s}^{\prime} / u_{e}$, plotted for the same streamwise locations as in figure 17. For each profile, the dashed vertical line indicate the zero value and the location where the profile was measured, while the small vertical segment indicates the value $u_{r m s}^{\prime} / u_{e}=0.1$. The first profile shows the characteristic peak in the buffer region of the incoming boundary layer. For $y / \delta_{0}<0$ the same profile shows a second weaker peak indicating the presence of a turbulent boundary layer flowing along the cell wall, emerging from the cusp and merging with the outer flow. This first region of the shear layer is characterised by very intense intermittent velocity fluctuations, with a flat- ness of the order of 40 and a positive skewness of the order of 4 . In fact, this region of the flow is characterised by large velocity gradients with the associated large values of turbulent kinetic energy production.

The cell flow is characterised by quite weak turbulence activity, with values of $u_{r m s}^{\prime} / u_{e}$ on the order of 0.015 in the region $-0.5<y / \delta_{0}<-0.1$, and for $x / L<0.4$. These values are slightly lower than what observed by Hokpunna and Manhart (2007), despite the mean velocities are in better agreement in the same region. As the shear layer evolves downstream, the peak value of $u_{r m s}^{\prime} / u_{e}$ remains essentially unchanged from the initial value 0.12 . However, a significant spreading of the region of intense fluctuations is observed, especially towards the cavity interior, as also the position of the peak of $u_{r m s}^{\prime} / u_{e}$ moves slightly in this direction in the first half of the shear layer.

More intense fluctuations, but only larger by a 20 to $30 \%$ than that of the peak in the upstream boundary layer, are observed near the shear layer impingement region. As the shear layer reattaches on the downstream part of the cell, the turbulent fluctuations peak lifts up and remains detached from the wall, at a normalised distance of about 0.25 . Interestingly, this area is the same associated to the velocity deficit observed in the corresponding profiles of $\bar{u} / u_{e}$ in figure 17 . Furthermore, a second peak of $u_{r m s}^{\prime} / u_{e}$, related to the buffer region of the turbulent boundary layer developing downstream of the cell, appears near the wall in the last three profiles.

The rate of spreading of the turbulent shear layer for case $\mathrm{C} 2$ is presented in figure 19, where the evolution of the shear layer vorticity thickness $\delta_{\omega}$ is plotted against the streamwise coordinate $x / L$. The vorticity thickness increases much more rapidly than in case C1, mainly because of the very large velocity gradients that characterise the shear layer in the first region of the shear layer and the high turbulent mixing. The vorticity thickness increases with an initial slope $\mathrm{d} \delta_{\omega} / \mathrm{d} x$ of about 0.41 , but the slope of the curve decreases as the shear layer evolves downstream.

\subsubsection{Spectral analysis}

Figure 20 shows the power spectral density of the normalised velocity fluctuations at three selected locations in the flow. The locations are: point $A$, in the middle of the shear layer region, at $x / L=0.5$ and at a $y / \delta_{0}=0$; point $B$, located inside the cavity at $y / \delta_{0}=-0.68$ at the same streamwise coordinate as for point $A$; and point $C$, located at $x / L=2.05$ and $y / \delta_{0}=0.091$, far downstream of the cell and close to the wall, below the 


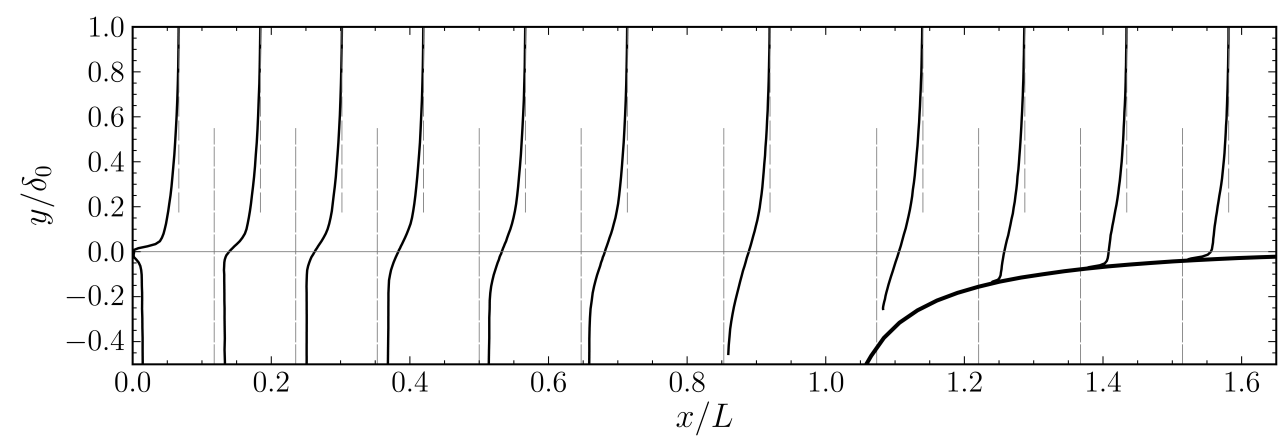

Fig. 17 Profiles of $\bar{u}(y) / u_{e}$ at selected locations along the shear layer. The downstream cavity shoulder is also visible on the right.

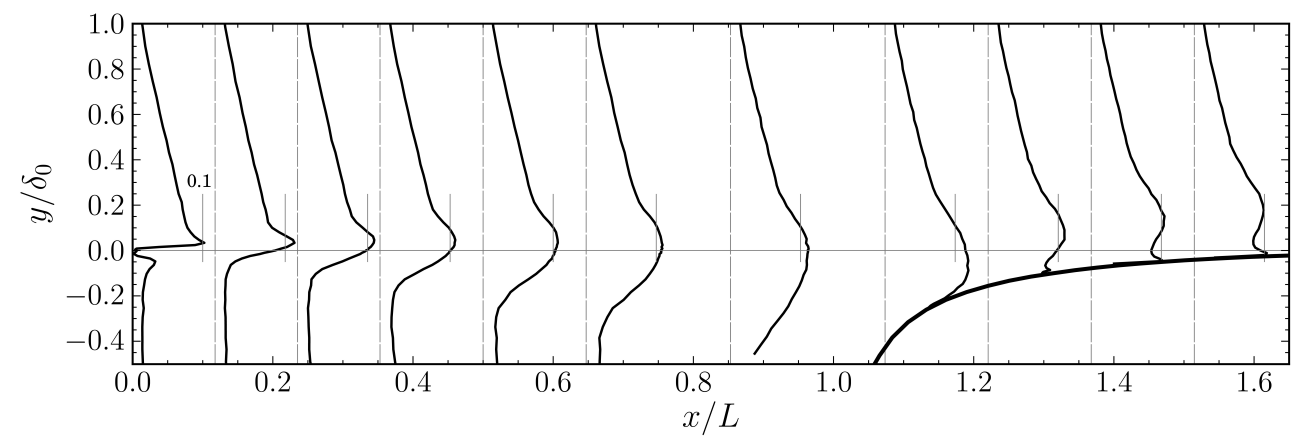

Fig. 18 Profiles of $u_{r m s}^{\prime}(y) / u_{e}$ at several selected locations along the shear layer.

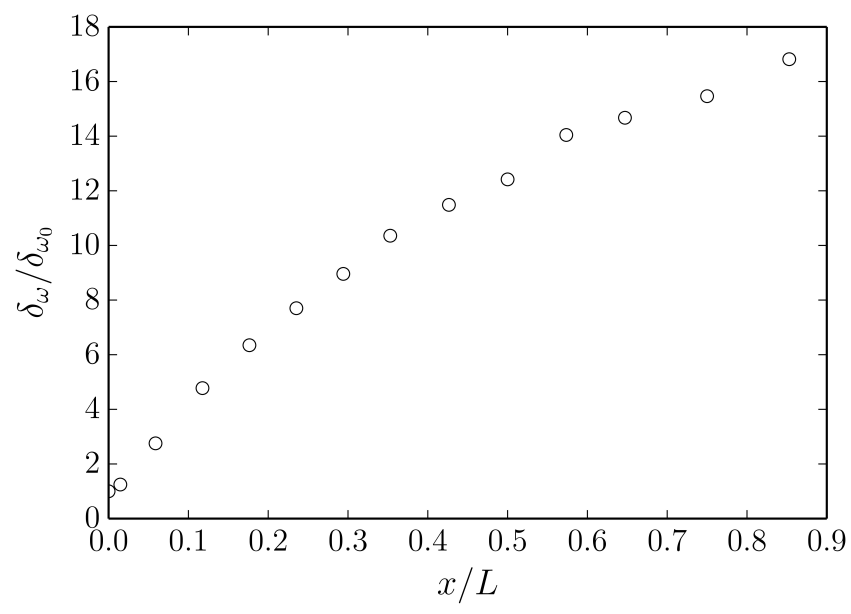

Fig. 19 Streamwise evolution of the non-dimensional vorticity thickness.

region of the velocity defect shown in the mean velocity profiles of figure 17.

The spectra are reported in a linear-linear scale and only the low frequency range is shown in order to better highlight its most significant feature. The three spectra are characterised by a low frequency mode at $7 \mathrm{~Hz}$, corresponding to a Strouhal number $S t=f L / u_{e}=0.038$. The peak at this frequency is more intense for location $B$ located inside the cavity, whereas for point $C$,

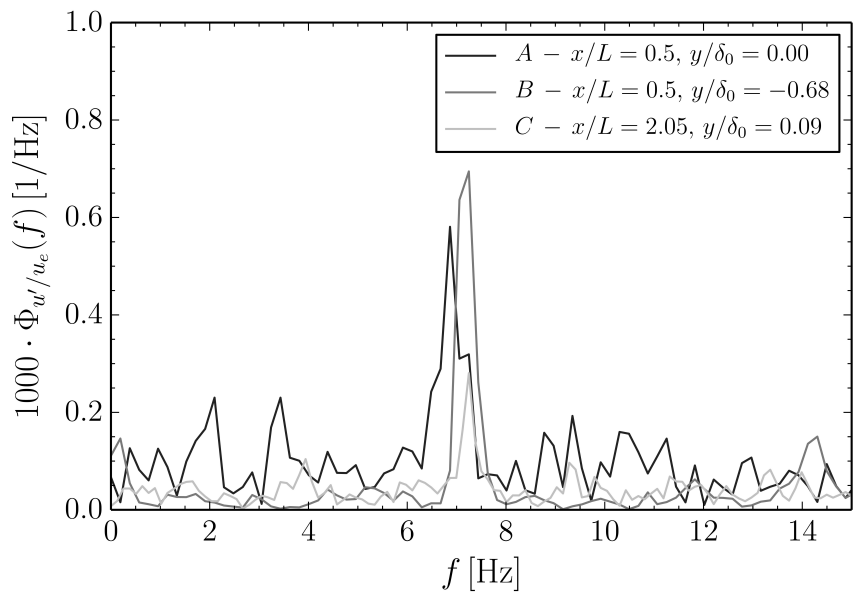

Fig. 20 Power spectral density of the normalised velocity fluctuations $u^{\prime} / u_{e}$ at three selected locations in the flow.

located further downstream in the turbulent boundary layer, a weak signature of the same phenomenon can still be observed.

It has to be remarked that this peak is the only significant feature of a spectrum which is otherwise characteristics of a turbulent flow. The turbulent shear layer is certainly not subject to self-sustained oscillations, due to the relatively large momentum thickness of the incoming boundary layer, that would in any case develop at frequencies much higher than $7 \mathrm{~Hz}$, at Strouhal num- 
ber of order one, and not two orders of magnitude lower. It is anticipated that the observed peak is associated with a large scale unsteadiness of the recirculating cell flow. Analysis of the individual spectral densities, not reported here, shows that this frequency increases quite slowly with the outer velocity.

Interestingly, the weak peak observed at point $C$ suggests that the vortex unsteadiness only affects the near wall region of the flow developing downstream of the cell. In fact, examination of all the velocity fluctuations spectra at varying distance from the wall at $x / L=2.05$ suggests that from $y / \delta_{0} \approx 0.2$ upwards, the signature of the low frequency mode cannot be detected any more, and is certainly not visible in the velocity defect region of the downstream boundary layer.

A short time history of the velocity signal in the cavity at location $B$ is reported in figure 21 . The signal is characterised by an alternating sequence of negative fluctuations, some short but intense, the other longer and weaker. The negative fluctuations are clearly separated by a period of about $0.13 \mathrm{~s}$, approximately $(7 \mathrm{~Hz})^{-1}$, i.e. the inverse of the low frequency mode. The total amplitude associated to this oscillation is on the order of 0.15 , which is quite significant for the the dynamics of the internal flow.

Wall-pressure fluctuations spectra are shown in figure 22. On each panel, the grey curve is the velocity fluctuation spectrum at $x / L=0.58, y / \delta_{0}=-1.1$, reported for reference with an artificial shift downwards of two decades.

A first feature is the significantly larger energy of the wall-pressure fluctuations measured near the shear layer impingement region by microphone $m_{2}$. In fact, the standard deviation of signal $p_{2}^{\prime}$ is $4.05 \mathrm{~Pa}$, about three times larger then for $p_{1}^{\prime}, p_{1 \mathrm{rms}}^{\prime}=1.45 \mathrm{~Pa}$, and $p_{3}^{\prime}$, $p_{3 r m s}^{\prime}=1.66 \mathrm{~Pa}$. These are conservative values since a large fraction of energy for the three signals is still concentrated around $f_{w t}$, as it is visible in the three panels. However, the peak energy is constant for the three signals since there is no pressure-velocity coupling enhancing the energy of the fluctuations at $f_{w t}$ for the two microphones inside the cavity, as it was observed in case $\mathrm{C} 1$.

The significantly larger energy for signal $p_{2}^{\prime}$ is due to the fact that the microphone is located in a highly turbulent region, where a wide range of small scale turbulent structures from the turbulent shear layer impinge on the wall. Furthermore, it is interesting to observe that the spectrum of signal $p_{2}^{\prime}$ is characterised by a distinct peak at $7 \mathrm{~Hz}$ a feature deriving from the dynamics of the cell flow, as opposed to the spectrum of microphone $m_{1}$, which does not show this peak.
The spectrum of $p_{3}^{\prime}$ is very similar to that of $p_{1}^{\prime}$ at low-medium frequencies, below $150 \mathrm{~Hz}$. This might suggests that the peaks in this range are not due to some relevant dynamics of the flow inside the cavity. By contrast, the spectrum of $p_{3}^{\prime}$ shows larger energies at higher frequencies, although not as high as for microphone $m_{2}$, due to the intense pressure fluctuations associated with the turbulent wall flow over microphone $m_{3}$. The pressure-velocity coupling is much weaker in case $\mathrm{C} 2$.

As an example, the cross-correlation coefficient function $\rho_{u^{\prime} p_{2}^{\prime}}(\tau)$, at $x / L=0.58, y / \delta_{0}=-1.1$, appears as in figure $23-(b)$. The cross-correlation is clearly periodic, with the periodicity given by the low frequency mode of the cell flow. By contrast, the cross correlation function for signal $p_{1}^{\prime}$, for the same velocity measurements, in figure 23- $(a)$, does not exhibit any clear periodicity as for $p_{2}^{\prime}$, likely because the signature of the low-frequency oscillation on this pressure signal is too weak, and similarly for $p_{3}^{\prime}$.

A possible explanation for the stronger correlation between the velocity fluctuations in the cell flow and the wall-pressure fluctuations in the impingement region for signal $p_{2}^{\prime}$ is that the low-frequency oscillation of the cavity flow induces a low-frequency flapping of the shear layer. This phenomenon produces on the impingement region an intense fluctuation of the pressure on microphone $m_{2}$, due to the highly energetic fluid in the shear layer.

Low frequency modes in open cavities have been previously observed in square-section geometries. The so called "wake mode", Gharib and Roshko (1987); Rowley et al (2001), is a large scale unsteadiness of the entire cavity flow, associated with violent and periodic ejections of vorticity, similarly to vortex shedding past a bluff-body. In their studies on vortex cores in large-aspect-ratio cylindrical cells, Savelsberg and Castro (2008), the cell flow was essentially steady, despite the presence of a spanwise instability. No low-frequency periodicities were observed, although it is important to note that the entire shear layer was subject to a favourable pressure gradient, due to the particular geometry of the problem.

\subsubsection{Effects on boundary layer}

In figure 24 the mean velocity profiles, panel $(a)$, and the profiles of the root-mean-square value of the velocity fluctuations, panel $(b)$, of the boundary layers at the reference upstream and downstream locations are compared. The mean velocity profile downstream of the cell shows a marked velocity deficit from $y / \delta_{0}>0.1$ up to the outer layers, with a major depression at around 


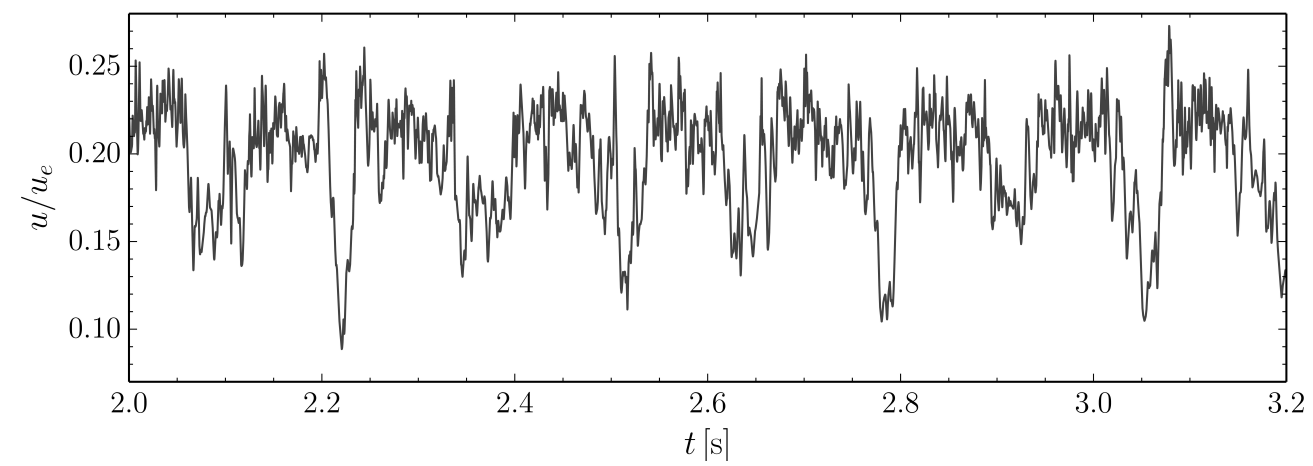

Fig. 21 Example of velocity time history at control point $B$, located inside the cavity at $x / L=0.5, y / \delta_{0}=-0.68$.

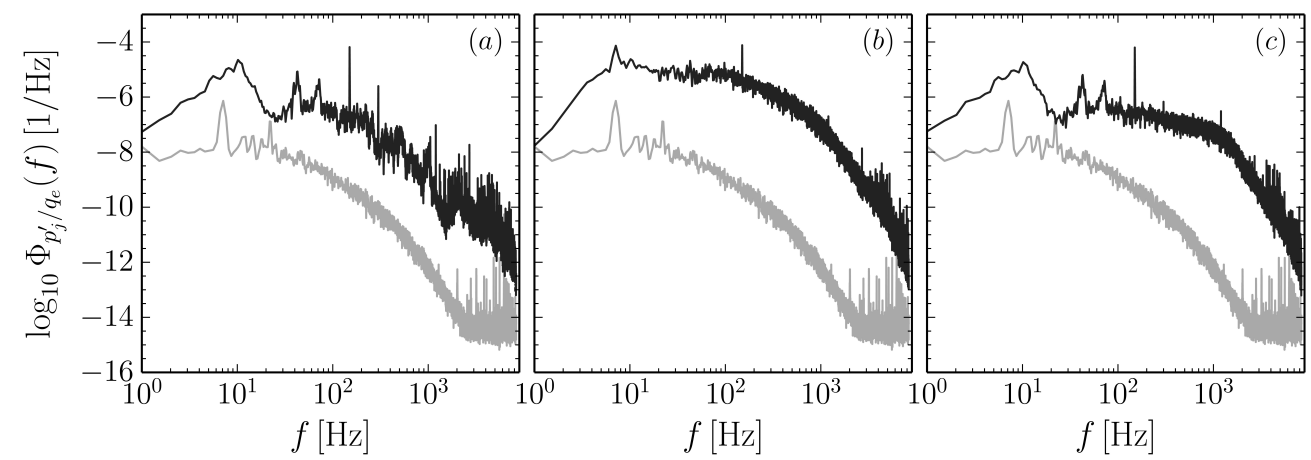

Fig. 22 Pressure fluctuation spectra for three representative microphones, (see figure 1). The grey curve is the velocity spectrum at $x / L=0.58, y / \delta_{0}=-1.1$, reported for reference over an artificial vertical scale.
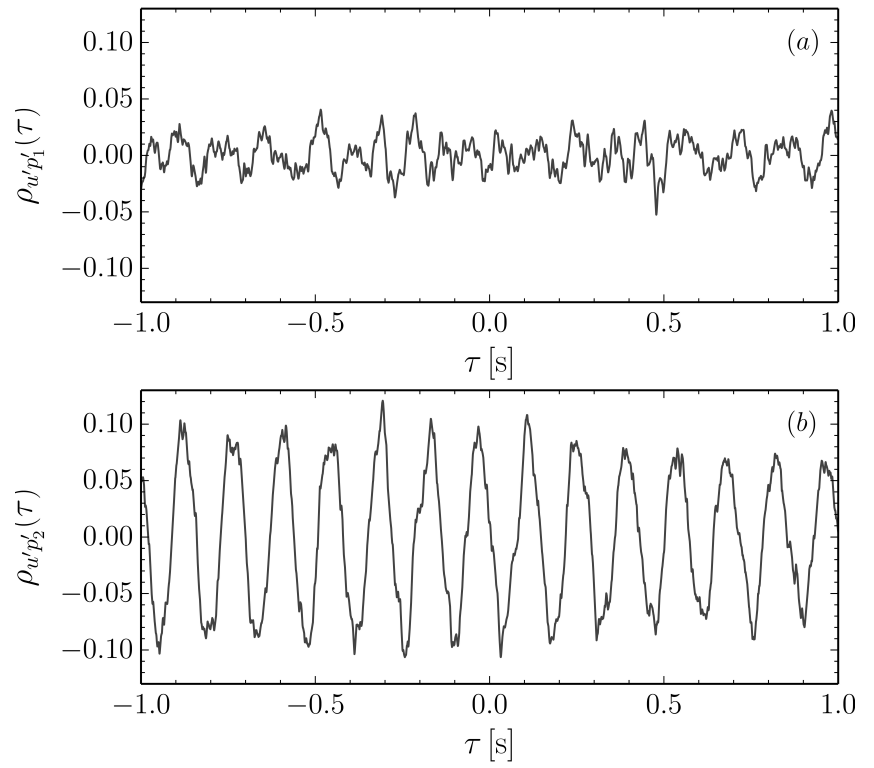

Fig. 23 Cross-correlation coefficient function $\rho_{u^{\prime} p_{j}^{\prime}}(\tau)$, with $u^{\prime}$ measured inside the cavity region, $x / L=0.58, y / \delta_{0}=$ -1.1 , and for the wall-pressure signals $p_{1}^{\prime}$, part $(a)$, and $p_{2}^{\prime}$, part $(b)$.

$y / \delta_{0} \approx 0.3$. The momentum thickness varies from 2.05 $\mathrm{mm}$, at $x / L=-0.14$, to $2.53 \mathrm{~mm}$, at $x / L=2.05$. Crucially, even without self-sustained oscillations, the increase is larger than what would occur if the cell had been replaced with a flat solid wall. In fact, assuming conservatively a constant skin friction coefficient between the two locations, equal to the value estimated at the first section, see table 1 , and using the boundary layer integral equation, the momentum thickness at the same downstream location would be $2.34 \mathrm{~mm}$. This momentum deficit is due to the transfer of momentum towards the cell flow, needed to sustain the rotation of the vortical structure, against the dissipation in the core and the drag associated to the internal boundary layer.

The profiles in figure 24 provide insight into the origin of the deficit in the mean velocity profile downstream of the cavity. In fact, the velocity depression is associated to a significant large region of high turbulence intensity, extending from $y / \delta_{0} \approx 0.1$ up to the outer flow, peaking at around $y / \delta_{0} \approx 0.3$. By observation of the profiles in figure 18 , this region can be associated to the highly turbulent flow developing in the central part of the shear layer, which first moves towards the interior of the cavity, for $x / L<1.0$ and which is then lifted upwards after impinging on the downstream part of the cell. Although not shown here for the sake of brevity, spectral analyses indicate that the increase of $u_{r m s}^{\prime} / u_{e}$ at a distance from the wall of about $y / \delta_{0}=0.3$ is broadband in nature, with higher energy across the entire spectrum. 

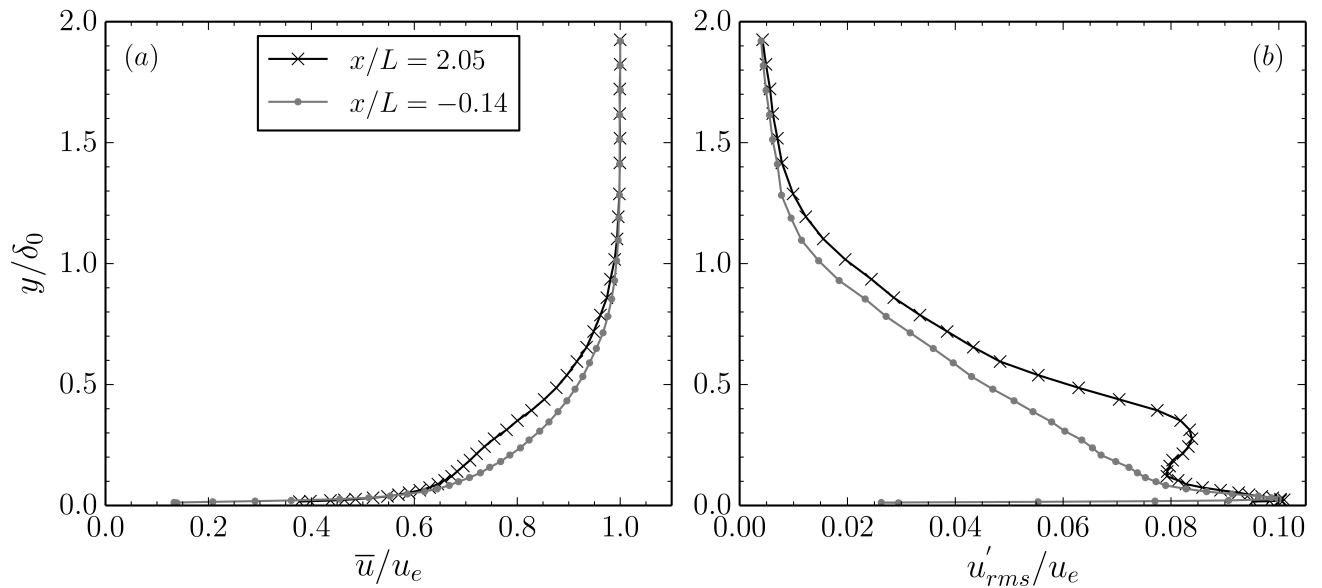

Fig. 24 Profiles of mean velocity, (a), and of the root-mean-square value of the velocity fluctuations, (b), upstream and downstream of the cell.

\subsection{Flow visualisations}

Smoke flow visualisations were performed for case $\mathrm{C} 2$ with the objective of shedding light over the low frequency mode. In figure 25 a sequence of six snapshots is shown. The snapshots are separated by $3 / 125 \mathrm{~s}$ and capture almost one complete cycle of the low frequency mode. The characteristic time $L / u_{e}$ is $0.55 \mathrm{~ms}$. The images appear blurred due to the relatively high exposure time, necessary to avoid under-exposure. As for case $\mathrm{C} 1$, the images were post-processed by subtraction of the mean image and by black/white equalisation. Inspection of the entire animated sequence of snapshots, available online as supplementary material, shows the vortex centre moving in a circular counter-clockwise motion around the geometric centre of the cell, that is in a direction opposite to the clockwise rotation of the flow. This phenomenon appears dynamically quite relevant for the internal flow as, the total displacement of the vortex centre is as large as $D / 2$. This value is much large than the vortex centre displacement that was predicted by Tutty et al (2012). These authors showed that the flow in a cylindrical vortex cell is susceptible to a steady inviscid spanwise mode that leads to a sinusoidal small displacement of the vortex core from the axis. In the present case, however, this feature is strongly unsteady. This large scale motion is responsible of the oscillatory features of the velocity signal in figure 21 . In fact, it is likely that the negative fluctuations in the time history are due to the low-speed vortex core passing over the hot wire probe.

As for case $\mathrm{C} 1$, in case $\mathrm{C} 2$ the erratic injection of high-energy fluid packets from the impingement region downwards into the cell can be observed clearly. These packets enter into the cell and drive the rotation of the vortex. We argue that this phenomenon is a characteris-
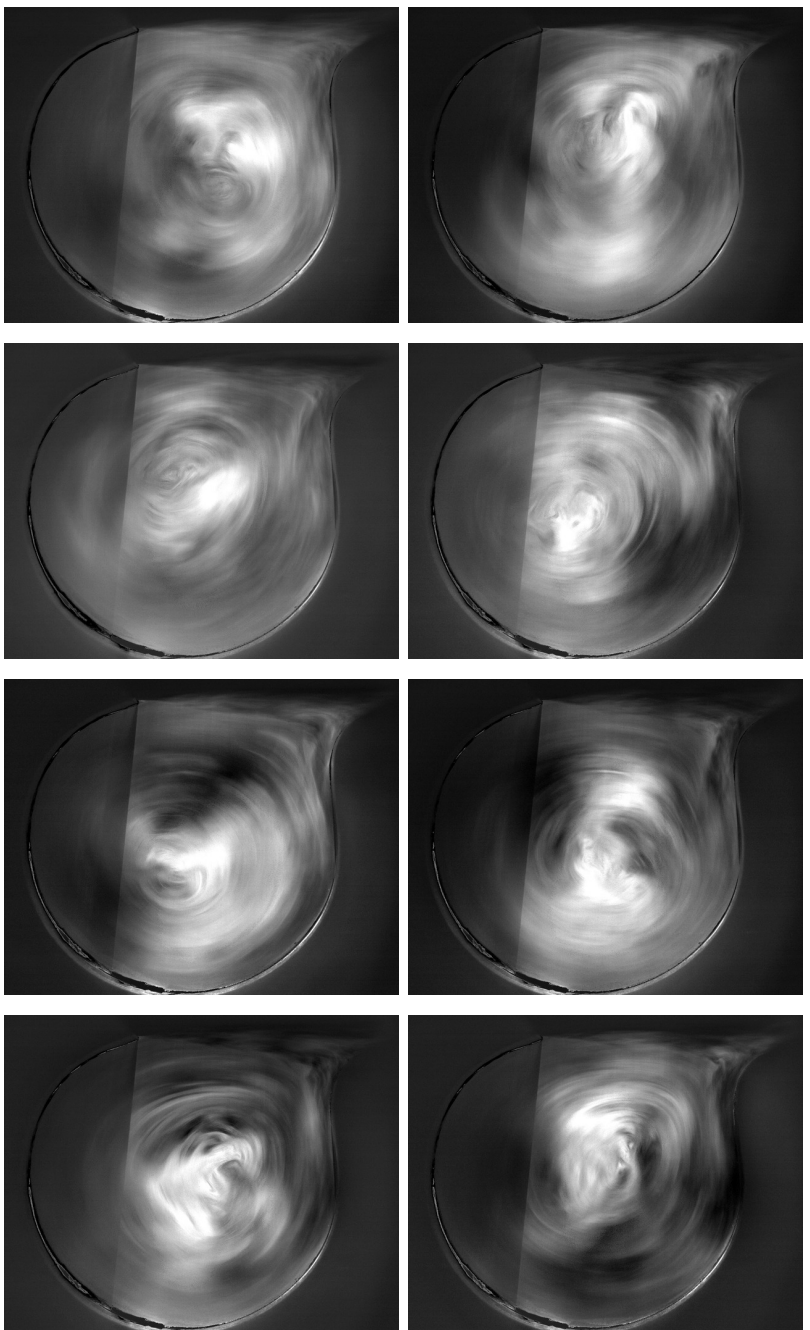

Fig. 25 Sequence of snapshots for case C2, sorted left to right, top to bottom. Time interval between successive frames is $3 / 125 \mathrm{~s}$. A supplementary movie is available online. 

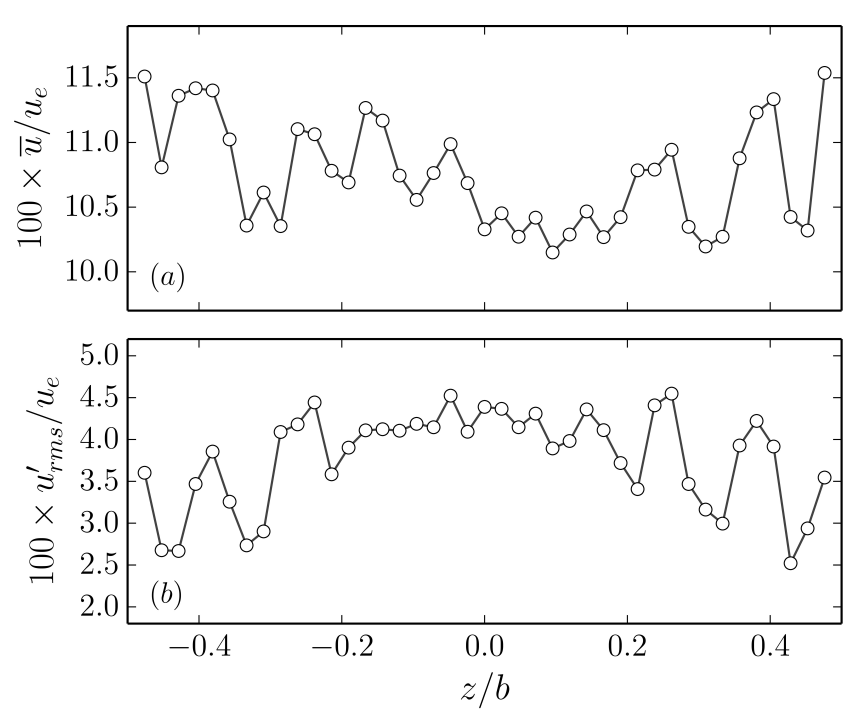

Fig. 26 Spanwise profiles in the cavity region at $x / L=$ $0.58, y / \delta_{0}=-1.36$. Mean velocity, $(a)$, and root-mean-square value of the velocity fluctuations, $(b)$.

tic feature of this type of geometry, probably connected to the particular shape of the downstream part of the cell. However, it is currently not understood if this phenomenon couples to or even drives the low-frequency oscillation of the vortex core. Finally, the flow visualisation also indicates that no large scale internal flow separation occurs at this Reynolds number, where the turbulence intensities in the internal boundary layer are certainly larger than in case C1.

\subsection{Three dimensional structure of the flow}

Figure 26 shows spanwise profiles of the mean velocity $\bar{u}(z) / u_{e}$, part $(a)$, and of the root-mean-square value of the velocity fluctuations $u_{r m s}^{\prime}(z) / u_{e}$, part $(b)$, measured in the interior of the cavity region, at $x / L=0.58$ and $y / \delta_{0}=-1.36$. This depth is higher than the thickness of the turbulent shear layer and, therefore, these profiles are representative of the structure of the internal cavity flow. The mean velocity profile is characterised by a distinct periodic pattern which affects the whole cavity, significantly enhanced at the two ends. The total amplitude of the mean velocity oscillation is quite small, on the order of 0.015. However, when compared to the spatial spanwise average it accounts for as much as $15 \%$. The spanwise wavelength $\lambda_{p}$ of this pattern can be estimate only roughly, but a value of $\lambda_{p} / b \approx 0.15$ seems reasonable. This value corresponds to a non-dimensional wavelength based on the cavity diameter equal to $\lambda_{p} / D=0.55$.

The profile of $u_{r m s}^{\prime}(z)$ clearly evidences the same peculiar pattern, mostly in the two extreme thirds of

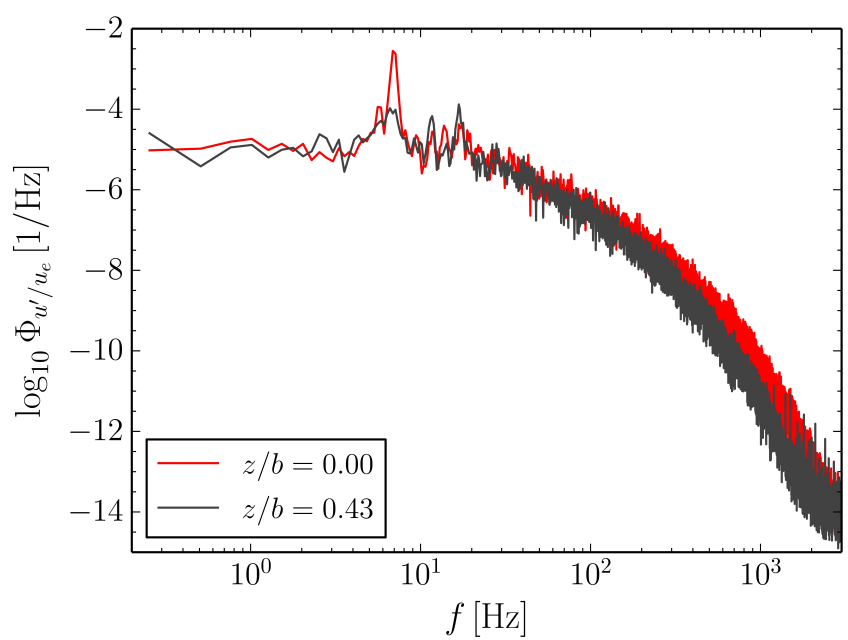

Fig. 27 Power spectral densities of the velocity fluctuations in the mid-span, $z / b=0.0$ lighter line, and at $z / b=0.43$, darker line, where $u_{r m s}^{\prime} / u_{e}$ is minimum.

the cavity span, for $z / b>0.2$ and for $z / b<-0.2$. The pattern consist of an alternation of regions of high and low value of $u_{r m s}^{\prime} / u_{e}$, with a peak-to-peak variation of the order of $0.02 u_{e}$. This pattern disappears in the central part of the cavity, where the value is essentially the same as for the peak in the lateral region.

Two velocity power spectra are shown in figure 27 , for two spanwise locations, $z / b=0.0$ and $z / b=0.43$, the position where $u_{r m s}^{\prime} / u_{e}$ is minimum. The difference in total energy between the velocity signals at the two control locations is essentially due to the low-frequency oscillation of the vortex flow. In fact, at the lateral position the peak is at least one decade less intense than in the mid span. Further differences are observed at higher frequencies, $100<f<2000 \mathrm{~Hz}$, but due to the logarithmic scale, they have less impact on the total variance of the signals. However, this feature seems also to suggest a slightly different structure of the turbulence at the two locations. This result seems to suggest that the spanwise modulation of $u_{r m s}^{\prime} / u_{e}$ is associated to a modulation of the oscillatory motion of the cavity flow.

The present results share some similarity with the structure observed in Faure et al (2006) and Faure et al (2009), in a square open cavity with large aspect ratio at Reynolds numbers between 40000 and 90000, as the wavelength was about half the cavity size, although the flow in those experiments was laminar or transitional and not highly turbulent as in the present case. In the present experiments the three dimensional character of the flow is stronger near the end walls, and seems to loose strength towards the centre. In other works, e.g. in Albensoeder et al (2001) for lid-driven cavities, the end walls have the opposite effect and suppress the three-dimensionality away from the midspan. 
By contrast, the three-dimensional steady flow modulation in large-aspect ratio vortex cells, a geometry that more closely resembles the present and investigated by Savelsberg and Castro (2008), had a wavelength that is about twice the cell diameter. This wavelength is almost four times larger than that found in the present experiments, and Reynolds number effects do not appear to be so important as the spanwise modulation observed by Savelsberg and Castro (2008) is quite robust to variations of the Reynolds number over an order of magnitude. Similarly, Maull and East (1963) examined the turbulent flow in a cylindrical open cavity and found a cellular spanwise structure with a wavelength about twice the cavity diameter. Further work is needed to elucidate in better detail this feature.

\section{Discussions and Conclusions}

In this paper we have reported experimental results of an investigation on the flow features in a trapped vortex cell (TVC). A configuration where the TVC is embedded into a flat plate and interacts with a zero-pressuregradient boundary layer has been studied. Hot-wire anemometry, wall-pressure fluctuation measurements and smoke flow visualisations were performed and reported for two very different and representative flow regimes.

In the first regime, at a moderate Reynolds number, the upstream boundary layer is laminar and its momentum thickness is small with respect to the cavity opening length. In such a condition the TVC displays strong self-sustained oscillations, resulting from a lock-in with a wind tunnel resonance. Smoke flow visualisations suggest that the driving mechanisms of the weakly rotating vortex is the erratic injection of high kinetic energy packets from the downstream edge of the cell, rather than the inefficient viscous momentum transfer through the laminar shear layer. The strong linear amplification of the velocity fluctuations in the shear layer, and the break up into turbulence of the coherent structures originated in this region, produces a turbulent boundary layer downstream. This boundary layer is significantly thicker than that upstream and carries the clear signature of the dissipation encountered along the shear layer evolution, visible as a marked velocity defect.

The second regime, at a moderately higher Reynolds number, is characterised by a turbulent incoming boundary layer whose momentum thickness is much larger than in the first regime. In this condition, due to the lower value of the ratio $L / \theta_{0}$, the shear layer does not develop strong self-sustained oscillations. Nevertheless, a comparison of the mean velocity profiles in the boundary layer between two reference locations upstream and downstream of the cavity clearly indicates a marked velocity defect in the boundary layer downstream, a symptom of a loss of momentum of the fluid flowing past the cell. This velocity defect has been associated to the momentum transfer operated by the turbulent stresses which sustain the rotation of the vortex flow, much faster than in the first condition. A further important flow feature of this regime is the presence of a low frequency modulation of the entire cell flow, consisting in a motion of the centre of the vortex core. This feature is associated to a spanwise modulation of the internal flow and share similarities with the spanwise instability found by other investigators, e.g. Tutty et al (2012), Hokpunna and Manhart (2007), in a similar circular geometry, although the spanwise wavelength found in the present experiments in about one fourth of that observed by these authors.

Finally, our investigations show that the increase in momentum thickness across the cell, a parameter that we used to quantify the global effects of the cell on the incoming boundary layer, is larger than what would naturally occur if the cell had been replaced by a flat solid wall, for both regimes discussed, where the flow physics are significantly different. The present experiments do not allow extending our conclusions to high-Reynolds flight conditions. However, the trends observed here might shed light on recent experimental investigations on passive control on airfoils with trapped vortex cells, where poor aerodynamic performance was observed, and mainly attributed to unsteady flow phenomena. The present results seem to indicate that successful control implementations with TVCs should take in strong consideration the dynamic of the shear layer, in particular if self-sustained oscillations are expected. In fact, this phenomenon provides an effective way to extract energy fom the mean flow, which is then ultimately dissipated, resulting in a performance loss. By contrast, the dynamics of the internal cavity flow, with a strong three dimensional character, do not appear to have a marked effect on the characteristics of the boundary layer downstream of the cell.

\section{References}

Adkins RC (1975) A short diffuser with low pressure loss. J Fluids Eng 97:297-302

Albensoeder S, Kuhlmann HC, Rath HJ (2001) Threedimensional centrifugal-flow instabilities in the liddriven-cavity problem. Phys Fluids 13(1):121-135

Baranov PA, Guvernyuk SV, Zubin MA, Isaev SA (2000) Numerical and physical modeling of the circulation flow in a vortex cell in the wall of a rectilinear channel. Fluid Dyn 35(5):663-673 
Basley J, Pastur LR, Lusseyran F, Faure TM, Delprat N (2010) Experimental investigation of global structures in an incompressible cavity flow using timeresolved PIV. Exp Fluids 50(4):905-918

Bendat J, Piersol A (1986) Random data: analysis and measurement procedures. John Wiley \& Sons

Brès G, Colonius T (2008) Three-dimensional instabilities in compressible flow over open cavities. J Fluid Mech 599:309-339

Bunyakin AV, Chernyshenko SI, Stepanov GY (1998) High-Reynolds-number Batchelor-model asymptotics of a flow past an aerofoil with a vortex trapped in a cavity. J Fluid Mech 358(1):283-297

Chatellier L, Laumonier J, Gervais Y (2004) Theoretical and experimental investigations of low Mach number turbulent cavity flows. Exp Fluids 36(5):728-740

Chernyshenko S, Galletti B, Iollo A, Zannetti L (2003) Trapped vortices and a favorable pressure gradient. J Fluid Mech pp 235-255

Chernyshenko SI, Castro IP, Hetsch T, Iollo A, Minisci E, Savelsberg R (2008) Vortex cell shape optimization for separation control. In: 8th ECCOMAS, European Congress on Computational Methods in Applied Sciences and Engineering, 30 June - 4 July 2008, Venice, Italy

Choudhry A, Arjomandi M, Kelso R (2016) Methods to control dynamic stall for wind turbine applications. Renewable Energy 86:26-37

De Gregorio F, Fraioli G (2008) Flow control on a high thickness airfoil by a trapped vortex cavity. In: 14th International Symposium on Applications of Laser Techniques to Fluid Mechanics, 7-10 July 2008, Lisbon, Portugal

Donelli R, Chernyshenko S, Iannelli P, Iollo A, Zannetti L (2009a) Flow models for a vortex cell. AIAA J 2(47):451-467

Donelli RS, Iannelli P, Iuliano E, De Rosa D (2009b) Suction optimization on thick airfoil to trap vortices. In: XIX Congresso AIMETA, 14-17 September 2009, Ancona, Italy

Faure T, Pastur L, Lusseyran F, Fraigneau Y, Bisch D (2009) Three-dimensional centrifugal instabilities development inside a parallelepipedic open cavity of various shape. Exp Fluids 47(3):395-410

Faure TM (2014) Velocity field and parametric analysis of a subsonic, medium-reynolds number cavity flow. Exp Fluids 55(11):1-18

Faure TM, Adrianos P, Lusseyran F, Pastur L (2006) Visualizations of the flow inside an open cavity at medium range Reynolds numbers. Exp Fluids 42(2):169-184

Garcia-Sagrado A, Hynes T (2011) Stochastic estimation of flow near the trailing edge of a NACA0012 airfoil. Exp Fluids 51:1057-1071

Gharib M, Roshko A (1987) The effect of flow oscillations on cavity drag. J Fluid Mech 177:501-530

Hokpunna A, Manhart M (2007) A large-eddy simulation of vortex cell flow with incoming turbulent boundary layer. Int J Mech Syst Sci Eng 1:123-128

Kasper W (1974) Aircraft wing with vortex generation. U.S Patent No. 3831885

Kuhlmann HC, Wanschura M, Rath HJ (1997) Flow in two-sided lid-driven cavities: non-uniqueness, instabilities, and cellular structures. J Fluid Mech 336:267-299

Kuo Ch, Huang Sh (2001) Influence of flow path modification on oscillation of cavity shear layer. Exp Fluids 31(2):162-178

Larchevêque L, Sagaut P, Labbé O (2007) Largeeddy simulation of a subsonic cavity flow including asymmetric three-dimensional effects. J Fluid Mech 577:105-126

Lasagna D, Donelli R, De Gregorio F, Iuso G (2011) Effects of a trapped vortex cell on a thick wing airfoil. Exp Fluids 51(5):1369-1384

Lasagna D, Orazi M, Iuso G (2013) Multi-time delay, multi-point linear stochastic estimation of a cavity shear layer velocity from wall-pressure measurements. Phys Fluids (1994-present) 25(1):017,101

Lawson SJ, Barakos GN (2011) Review of numerical simulations for high-speed, turbulent cavity flows. Prog Aerosp Sci 47(3):186-216

Mariotti A, Grozescu A, Buresti G, Salvetti M (2013) Separation control and efficiency improvement in a $2 \mathrm{D}$ diffuser by means of contoured cavities. Eur J Mech B-Fluid 41(0):138 - 149

Mariotti A, Buresti G, Salvetti M (2014) Control of the turbulent flow in a plane diffuser through optimized contoured cavities. Eur J Mech B-Fluid 48(0):254 265

Maull DJ, East LF (1963) Three-dimensional flow in cavities. J Fluid Mech 16:620-632

Olsman W, Willems J, Hirschberg A, Colonius T, Trieling R (2011) Flow around a NACA0018 airfoil with a cavity and its dynamical response to acoustic forcing. Exp Fluids 51(2):493-509

Ringleb FO (1961) Separation control by trapped vortices. In: Boundary Layer and flow control, Pergamon Press, Ed. Lachmann G.V.

Rockwell D, Knisely C (1980) Observations of the threedimensional nature of unstable flow past a cavity. Phys Fluids 23:425-431

Rockwell D, Naudascher E (1978) Review-selfsustaining oscillations of flow past cavities. J Fluid Eng 100(2):152-165 
Rowley C, Colonius T, Basu A (2001) On self-sustained oscillations in two dimensional compressible flow over rectangular cavities. J Fluid Mech 455:315-345

Savelsberg R, Castro I (2008) Vortex flows in open cylindrical-section cavities. Exp Fluids 46(3):485-497

Savitsky A, Schukin L, Kareljn V (1995) Method for control of the boundary layer on the aerodynamic surface of an aircraft, and the aircraft provided with boundary layer control system. U.S Patent No. 5417391

Tutty O, Savelsberg R, Castro IP (2012) Threedimensional flow in circular cavities of large spanwise aspect ratio. J Fluid Mech 707:551-574

Tutty O, Buffoni M, Kerminbekov R, Donelli R, De Gregorio F, Rogers E (2013) Control of flow with trapped vortices: theory and experiments. Int J Flow Control 5(2):89-110

de Vicente J, Basley J, Meseguer-Garrido F, Soria J, Theofilis V (2014) Three-dimensional instabilities over a rectangular open cavity: from linear stability analysis to experimentation. J Fluid Mech 748:189220

Vuddagiri A, Samad A (2013) Vortex trapping by different cavities on an airfoil. Wind Eng 37(5):469-482

Zannetti L (2006) Vortex equilibrium in flows past bluff bodies. J Fluid Mech 562:151-171

Zhang K, Naguib AM (2011) Effect of finite cavity width on flow oscillation in a low-Mach-number cavity flow. Exp Fluids 51(5):1209-1229 